\title{
Local and global well-posedness for the 2D Zakharov-Kuznetsov-Burgers equation in low regularity Sobolev space
}

\author{
Hiroyuki Hirayama \\ Organization for Promotion of Tenure Track, University of Miyazaki, \\ Miyazaki, 889-2192, Japan \\ E-mail address, h.hirayama@cc.miyazaki-u.ac.jp
}

\begin{abstract}
In the present paper, we consider the Cauchy problem of the $2 \mathrm{D}$ Zakharov-Kuznetsov-Burgers (ZKB) equation, which has the dissipative term $-\partial_{x}^{2} u$. This is known that the $2 \mathrm{D}$ Zakharov-Kuznetsov equation is well-posed in $H^{s}\left(\mathbb{R}^{2}\right)$ for $s>1 / 2$, and the $2 \mathrm{D}$ nonlinear parabolic equation with quadratic derivative nonlinearity is well-posed in $H^{s}\left(\mathbb{R}^{2}\right)$ for $s \geq 0$. By using the Fourier restriction norm with dissipative effect, we prove the well-posedness for ZKB equation in $H^{s}\left(\mathbb{R}^{2}\right)$ for $s>-1 / 2$.

Key Words and Phrases. Zakharov-Kuznetsov equation, Burgers equation, well-posedness, Cauchy problem, Fourier restriction norm.

2010 Mathematics Subject Classification. 35Q53.
\end{abstract}

\section{Introduction}

We consider the Cauchy problem of the 2D Zakharov-Kuznetsov-Burgers (ZKB) equation:

$$
\left\{\begin{array}{l}
\partial_{t} u+\partial_{x}\left(\partial_{x}^{2}+\partial_{y}^{2}\right) u-\partial_{x}^{2} u=\partial_{x}\left(u^{2}\right), \quad t>0,(x, y) \in \mathbb{R}^{2}, \\
u(0, x, y)=u_{0}(x, y), \quad(x, y) \in \mathbb{R}^{2},
\end{array}\right.
$$

where the unknown function $u$ is $\mathbb{R}$-valued. This equation is two dimensional model of the Kowteweg-de Vries-Burgers (KdVB) equation

$$
\partial_{t} u+\partial_{x}^{3} u-\partial_{x}^{2} u=\partial_{x}\left(u^{2}\right), \quad t>0, x \in \mathbb{R},
$$

and appears in the dust-ion-acoustic-waves in dusty-plasmas (See, 22, 24]). We can see that (1.1) has both dissipative term and dispersive term. The aim of this paper is to prove the well-posedness of (1.1) in the Sobolev space $H^{s}\left(\mathbb{R}^{2}\right)$.

First, we introduce some known results for related problems for $1 \mathrm{D}$ case. In [11, Kenig, Ponce, and Vega proved that the Kowteweg-de Vries (KdV) equation

$$
\partial_{t} u+\partial_{x}^{3} u=\partial_{x}\left(u^{2}\right), \quad t>0, x \in \mathbb{R},
$$

is locally well-posed in $H^{s}(\mathbb{R})$ for $s>-3 / 4$. Colliander, Keel, Stafillani, Takaoka, and Tao ([6]) extended the local result to globally in time. For the 
critical case, Kishimoto ([13) and Guo ([10]) obtained the global well-posedness of $\mathrm{KdV}$ equation in $H^{-\frac{3}{4}}(\mathbb{R})$. While, it is proved that the flow map of $\mathrm{KdV}$ equation is not uniformly continuous for $s<-3 / 4$ by Kenig, Ponce, and Vega in 12 ] (for $\mathbb{C}$-valued KdV) and Christ, Colliander, and Tao in [5] (for $\mathbb{R}$-valued KdV). Therefore, $s=-3 / 4$ is optimal regularity to obtain the well-posedness of KdV equation by using the iteration argument. For the Burgers equation

$$
\partial_{t} u-\partial_{x}^{2} u=\partial_{x}\left(u^{2}\right), \quad t>0, x \in \mathbb{R},
$$

Dix ([7]) proved the local well-posedness in $H^{s}(\mathbb{R})$ for $s>-1 / 2$ and nonuniqueness of solution for $s<-1 / 2$. For the critical case, Bekiranov ([2]) obtained the local well-posedness of the Burgers equation in $H^{-\frac{1}{2}}(\mathbb{R})$. These results say that $-1 / 2$ is optimal regularity to obtain the well-posedness of the Burgers equation. In [20, Molinet and Ribaud considered the KdV-Burgers equation

$$
\partial_{t} u+\partial_{x}^{3} u-\partial_{x}^{2} u=\partial_{x}\left(u^{2}\right), \quad t>0, x \in \mathbb{R}
$$

and obtained the global well-posedness in $H^{s}(\mathbb{R})$ for $s>-1$. For the critical case, Molinet and Vento (21]) proved the global well-posedness of the KdVBurgers equation in $H^{-1}(\mathbb{R})$. They also proved that the flow map is discontinuous for $s<-1$. We note that the regularity $s=-1$ is lower than both $-3 / 4$ and $-1 / 2$. It means that both the dispersive term and the dissipative term are essentially effective for well-posedness.

Next, we introduce some known results for related problems for 2D case. Grünrock and Herr ([9]), and Molinet and Pilod ([19]) proved that the 2D Zakharov-Kuznetsov equation

$$
\partial_{t} u+\partial_{x}\left(\partial_{x}^{2}+\partial_{y}^{2}\right) u=\partial_{x}\left(u^{2}\right), \quad t>0,(x, y) \in \mathbb{R}^{2}
$$

is locally well-posed in $H^{s}\left(\mathbb{R}^{2}\right)$ for $s>1 / 2$. Especially, Grünrock and Herr used the linear transform

$$
v(t, x, y)=u\left(t, \frac{4^{\frac{1}{3}}}{2}(x+y), \frac{4^{\frac{1}{3}}}{2 \sqrt{3}}(x-y)\right) .
$$

and rewrote (1.3) to the symmetric form

$$
\partial_{t} v+\left(\partial_{x}^{3}+\partial_{y}^{3}\right) v=4^{-\frac{1}{3}}\left(\partial_{x}+\partial_{y}\right)\left(v^{2}\right), \quad t>0, \quad(x, y) \in \mathbb{R}^{2} .
$$

Such transform is introduced by Artzi, Koch, and Saut in [1. We note that the well-posedness of (1.3) in $H^{s}\left(\mathbb{R}^{2}\right)$ is equivalent to the well-posedness of (1.4) in $H^{s}\left(\mathbb{R}^{2}\right)$. This transform is not essentially needed to obtain the well-posedness (Actually, Molinet and Pilod did not used such transform), but the symmetry helps us to find the structure of the equation and to write some parts of proof simply. Well-posedness of (1.3) for $s \leq 1 / 2$ is still open. But, Kinoshita gave the author the comment that there is a counter example for the $C^{2}$-well-posedness of (1.4) in $H^{s}\left(\mathbb{R}^{2}\right)$ for $s<-1 / 4$. His counter example is given as

$$
\widehat{u_{0}}(\xi, \eta):=N^{-s+\frac{5}{4}}\left(\chi_{A}(\xi, \eta)+\chi_{B}(\xi, \eta)\right),
$$


where

$$
\begin{aligned}
& A:=\left\{N a+N^{-\frac{1}{2}} \delta v+N^{-2} \epsilon \frac{v^{\perp}}{\left|v^{\perp}\right|} \mid-1<\delta, \epsilon<1\right\}, \\
& B:=\left\{N b+N^{-\frac{1}{2}} \delta v+N^{-2} \epsilon \frac{v^{\perp}}{\left|v^{\perp}\right|} \mid-1<\delta, \epsilon<1\right\}, \\
& v:=(3 \sqrt[3]{9}, \sqrt[3]{100}), a:=(\sqrt[3]{2}, \sqrt[3]{75}), b:=\left(-3 \sqrt[3]{2},-\frac{\sqrt[3]{75}}{5}\right) .
\end{aligned}
$$

Indeed, we can obtain $\left\|u_{0}\right\|_{H^{s}} \sim 1$ and

$$
\sup _{0<t \leq T}\left\|\int_{0}^{t} e^{-\left(t-t^{\prime}\right)\left(\partial_{x}^{3}+\partial_{y}^{3}\right)}\left(\partial_{x}+\partial_{y}\right)\left(\left(e^{-t^{\prime}\left(\partial_{x}^{3}+\partial_{y}^{3}\right)} u_{0}\right)^{2}\right) d t^{\prime}\right\|_{H^{s}} \gtrsim N^{-s-\frac{1}{4}} .
$$

While for the nonlinear parabolic equation

$$
\partial_{t} u-\Delta u=P(D) F(u), \quad t>0,(x, y) \in \mathbb{R}^{d},
$$

Ribaud ([23]) obtained some well-posedness results. His results contain that the well-posedness of the 2D nonlinear parabolic equation

$$
\partial_{t} u-\left(\partial_{x}^{2}+\partial_{y}^{2}\right) u=\partial_{x}\left(u^{2}\right), \quad t>0,(x, y) \in \mathbb{R}^{2}
$$

in $H^{s}\left(\mathbb{R}^{2}\right)$ for $s \geq 0$ and nonuniqueness of solution for $s<0$. Therefore, our interest is the well-posedness of (1.1) in $H^{s}\left(\mathbb{R}^{2}\right)$ for lower $s$ than both $-1 / 4$ and 0 .

Here, we introduce the results for $2 \mathrm{D}$ dispersive-dissipative models. The KP-Burgers equation

$$
\partial_{x}\left(\partial_{t} u+\partial_{x}^{3} u-\partial_{x}^{2} u-\partial_{x}\left(u^{2}\right)\right)+\epsilon \partial_{y}^{2} u=0, \quad t>0,(x, y) \in \mathbb{R}^{2}, \quad \epsilon \in\{-1,1\},
$$

is also two dimensional model of KdV-Burgers equation. We call KP-Burgers equation "KP-I-Burgers equation" if $\epsilon=-1$, and "KP-II-Burgers equation" if $\epsilon=1$. The well-posedness of KP-Burgers equation is obtained in $H^{s, 0}\left(\mathbb{R}^{2}\right)$ for $s>-1 / 2$ by Kojok in [14 (for $\epsilon=1$ ) and Mohamad in [18] (for $\epsilon=-1$ ). Where $H^{s, 0}\left(\mathbb{R}^{2}\right)$ is anisotropic Sobolev space defined by the norm $\|f\|_{H^{s, 0}}=$ $\left\|\langle\xi\rangle^{s} \widehat{f}(\xi, \eta)\right\|_{L_{\xi \eta}^{2}}$. Carvajal, Esfahani, and Panthee (4]) considered the two dimensional dissipative $\mathrm{KdV}$ type equation

$$
\partial_{t} u+\partial_{x}^{3} u+L_{x, y} u+\partial_{x}\left(u^{2}\right)=0, \quad t>0,(x, y) \in \mathbb{R}^{2},
$$

where the operator $L_{x, y}$ is defined by

$$
\mathcal{F}_{x y}\left[L_{x, y} f\right](\xi, \eta)=-\Phi(\xi, \eta) \widehat{f}(\xi, \eta)
$$

and the leading term of $\Phi(\xi, \eta)$ is $-\left(|\xi|^{p_{1}}+|\eta|^{p_{2}}\right)$ with $p_{1}, p_{2}>0$. They obtained the well-posedness of this equation with $p_{2}>1$ in $H^{s, 0}\left(\mathbb{R}^{2}\right)$ for $s>-3 / 4$. They also considered the high dimensional cases and obtained more general results. There is no results for the well-posedness of (1.1) as far as we know. But the initial-boundary problem of ZKB equation is studied by Larkin ([15, [16]). 
Now, we give the main results in this paper. To begin with, we rewrite (1.1) to the symmetric form based on [9]. We put

$$
v(t, x, y)=4 u\left(16 t, 2(x+y), 2 \sqrt{3}^{-1}(x-y)\right)
$$

Then, (1.1) can be rewritten

$$
\left\{\begin{array}{l}
\partial_{t} v+\left(\partial_{x}^{3}+\partial_{y}^{3}\right) v-\left(\partial_{x}+\partial_{y}\right)^{2} v=\left(\partial_{x}+\partial_{y}\right)\left(v^{2}\right) \\
v(0, x, y)=v_{0}(x, y):=4 u_{0}\left(2(x+y), 2 \sqrt{3}^{-1}(x-y)\right)
\end{array}\right.
$$

We note that the well-posedness of (1.1) in $H^{s}\left(\mathbb{R}^{2}\right)$ is equivalent to the wellposedness of (1.6) in $H^{s}\left(\mathbb{R}^{2}\right)$. Therefore, we consider (1.6) instead of (1.1).

Theorem 1.1. Let $s>-\frac{1}{2}$. Then (1.6) is locally well-posed in $H^{s}\left(\mathbb{R}^{2}\right)$. (Therefore (1.1) is also locally well-posed in $H^{s}\left(\mathbb{R}^{2}\right)$.) More precisely, for any $v_{0} \in H^{s}\left(\mathbb{R}^{2}\right)$, there exist $T>0$, and an unique solution $v \in X_{T}^{s, \frac{1}{2}, 1}(\hookrightarrow$ $C\left([0, T] ; H^{s}\left(\mathbb{R}^{2}\right)\right)$ (See, Definition [2.1) to (1.6) in $[0, T]$. Furthermore, the datato-solution map is Lipschitz continuous from $H^{s}\left(\mathbb{R}^{2}\right)$ to $C\left([0, T] ; H^{s}\left(\mathbb{R}^{2}\right)\right)$.

Theorem 1.2. Let $s>-\frac{1}{2}$. For any $v_{0} \in \widetilde{H}^{s}\left(\mathbb{R}^{2}\right)$, the solution $v$ obtained in Theorem 1.1 can be extended globally in time and $v$ belongs to $C\left((0, \infty) ; \widetilde{H}^{\infty}\left(\mathbb{R}^{2}\right)\right)$, where $\widetilde{H}^{s}\left(\mathbb{R}^{2}\right)$ is the completion of the Schwartz class $\mathcal{S}\left(\mathbb{R}^{2}\right)$ with the norm $\|f\|_{\widetilde{H}^{s}}=\left\|\langle\xi+\eta\rangle^{s} \widehat{f}(\xi, \eta)\right\|_{L_{\xi \eta}^{2}}$, and $\widetilde{H}^{\infty}\left(\mathbb{R}^{2}\right)=\bigcap_{s \in \mathbb{R}} \widetilde{H}^{s}\left(\mathbb{R}^{2}\right)$.

Remark 1.3. (i) Although (1.1) does not have the dissipative term with respect to $y$, the well-posedness of (1.1) is obtained in isotropic Sobolev space $H^{s}\left(\mathbb{R}^{2}\right)$ for lower regularity than both (1.3) and (1.5).

(ii) Theorem 1.2 says that (1.1) is globally well-posed in $H^{s, 0}\left(\mathbb{R}^{2}\right)$ for $s>-\frac{1}{2}$.

To obtain Theorem 1.1, we have to treat the dissipative term carefully, because the symbol $(\xi+\eta)^{2}$ is vanished on the line $\{(\xi,-\xi) \mid \xi \in \mathbb{R}\}$. But the nonlinear term is also vanished on the same line. It helps us to obtain the key bilinear estimate (Proposition 3.1). We will use the iteration argument with the Fourier restriction norm to obtain the local well-posedness. While, the global well-posedness will be proved by using the smoothing effect from the dissipative term and non-increasing of $L^{2}$-norm of the solution.

Notation. We denote the spatial Fourier transform by $\hat{\cdot}$ or $\mathcal{F}_{x y}$, the Fourier transform in time by $\mathcal{F}_{t}$, and the Fourier transform in all variables by $\sim$ or $\mathcal{F}$. The operator $U(t)=e^{-t\left(\partial_{x}^{3}+\partial_{y}^{3}\right)}$ and $W(t)=e^{|t|\left(\partial_{x}+\partial_{y}\right)^{2}} e^{-t\left(\partial_{x}^{3}+\partial_{y}^{3}\right)}$ on $H^{s}\left(\mathbb{R}^{2}\right)$ is given as a Fourier multiplier

$\mathcal{F}_{x y}[U(t) f](\xi, \eta)=e^{i t\left(\xi^{3}+\eta^{3}\right)} \widehat{f}(\xi), \quad \mathcal{F}_{x y}[W(t) f](\xi, \eta)=e^{-|t|(\xi+\eta)^{2}} e^{i t\left(\xi^{3}+\eta^{3}\right)} \widehat{f}(\xi)$.

$U(t)$ and $W(t)$ give a solution to

$$
\partial_{t} u+\left(\partial_{x}^{3}+\partial_{y}^{3}\right) u=0
$$

and

$$
\partial_{t} u+\left(\partial_{x}^{3}+\partial_{y}^{3}\right) u-\operatorname{sgn}(t)\left(\partial_{x}+\partial_{y}\right)^{2} u=0
$$

respectively. We note that $\mathcal{F}[U(-\cdot) F(\cdot)](\tau, \xi, \eta)=\widetilde{F}\left(\tau+\xi^{3}+\eta^{3}, \xi, \eta\right)$. 
We will use $A \lesssim B$ to denote an estimate of the form $A \leq C B$ for some constant $C$ and write $A \sim B$ to mean $A \lesssim B$ and $B \lesssim A$. We will use the convention that capital letters denote dyadic numbers, e.g. $N=2^{n}$ for $n \in \mathbb{Z}$ and for a dyadic summation we write $\sum_{N} a_{N}:=\sum_{n \in \mathbb{Z}} a_{2^{n}}, \sum_{N \geq N^{\prime}} a_{N}:=$ $\sum_{n \in \mathbb{Z}, 2^{n} \geq N^{\prime}} a_{2^{n}}$, and $\sum_{N \leq N^{\prime}} a_{N}:=\sum_{n \in \mathbb{Z}, 2^{n} \leq N^{\prime}} a_{2^{n}}$ for brevity. Let $\chi \in$ $C_{0}^{\infty}((-2,2))$ be an even, non-negative function such that $\chi(t)=1$ for $|t| \leq 1$. We define $\varphi(t):=\chi(t)-\chi(2 t)$ and $\varphi_{N}(t):=\varphi\left(N^{-1} t\right)$. Then, $\sum_{N} \varphi_{N}(t)=1$ whenever $t \neq 0$. We define the projections

$$
\begin{aligned}
& \widehat{P_{N} u}(\xi, \eta):=\varphi_{N}(|(\xi, \eta)|) \widehat{u}(\xi, \eta), \widehat{P_{N, M} u}(\xi, \eta):=\varphi_{N, M}(\xi, \eta) \widehat{u}(\xi, \eta), \\
& \widetilde{Q_{L} u}(\tau, \xi, \eta):=\varphi_{L}\left(\tau-\xi^{3}-\eta^{3}\right) \widetilde{u}(\tau, \xi, \eta),
\end{aligned}
$$

where $\varphi_{N, M}(\xi, \eta):=\varphi_{N}(|(\xi, \eta)|) \varphi_{M}(\xi+\eta)$.

The rest of this paper is planned as follows. In Section 2, we will give the definition of the solution space, and prove the linear estimates. In Section 3, we will prove the bilinear estimate which is main part of this paper. In Section 4, we will give the proof of the well-posedness (Theorems 1.1 and [1.2).

\section{Function space and linear estimate}

In this section, we define the function space, and prove the estimate for linear solution and Duhamel term. First, we consider the standard Fourier restriction norm $\|\cdot\|_{X^{s, b}}$ for (1.6) defined by

$$
\|u\|_{X^{s, b}}=\left\|\langle|(\xi, \eta)|\rangle^{s}\left\langle(\xi+\eta)^{2}+i\left(\tau-\xi^{3}-\eta^{3}\right)\right\rangle^{b} \widetilde{u}(\tau, \xi, \eta)\right\|_{L_{\tau \xi \eta}^{2}} .
$$

Such Fourier restriction norm was introduced by J. Bourgain ([3]) for the nonlinear Schrödinger equation and the $\mathrm{KdV}$ equation. Let $\psi \in C^{\infty}(\mathbb{R})$ denotes a cut-off function such that $\operatorname{supp} \psi \subset[-2,2], \psi=1$ on $[-1,1]$. We note that, the estimate

$$
\left\|\psi(t) W(t) u_{0}\right\|_{X^{s, b}} \lesssim\left\|\langle|(\xi, \eta)|\rangle^{s}\langle\xi+\eta\rangle^{b-\frac{1}{2} \widehat{u_{0}}}(\xi, \eta)\right\|_{L_{\xi \eta}^{2}}
$$

holds. Therefore, if $b \leq 1 / 2$, then $\psi W(\cdot) u_{0} \in X^{s, b}$ for $u_{0} \in H^{s}$. But the embedding $X^{s, b} \hookrightarrow C\left(\mathbb{R} ; H^{s}\left(\mathbb{R}^{2}\right)\right)$ does not hold for $b \leq 1 / 2$. Therefore, we use the Besov type Fourier restriction norm defined as follows.

Definition 2.1. Let $s \in \mathbb{R}, b \in \mathbb{R}$.

(i) We define the function space $X^{s, b, 1}$ as the completion of the Schwartz class $\mathcal{S}\left(\mathbb{R}_{t} \times \mathbb{R}_{x, y}^{2}\right)$ with the norm

$$
\|u\|_{X^{s, b, 1}}=\left\{\sum_{N \in 2^{\mathbb{Z}}} \sum_{M \in 2^{\mathbb{Z}}}\left(\sum_{L \in 2^{\mathbb{Z}}}\langle N\rangle^{s}\left\langle M^{2}+L\right\rangle^{b}\left\|P_{N, M} Q_{L} u\right\|_{L_{t x y}^{2}}\right)^{2}\right\}^{\frac{1}{2}} .
$$

(ii) For $T>0$, we define the time localized space $X_{T}^{s, b, 1}$ as

$$
X_{T}^{s, b, 1}=\left\{\left.u\right|_{[0, T]} \mid u \in X^{s, b, 1}\right\}
$$

with the norm

$$
\|u\|_{X_{T}^{s, b, 1}}=\inf \left\{\|v\|_{X^{s, b, 1}}\left|v \in X^{s, b, 1}, v\right|_{[0, T]}=\left.u\right|_{[0, T]}\right\} .
$$


Remark 2.2. (i) The embedding $X_{T}^{s, \frac{1}{2}, 1} \hookrightarrow C\left([0, T] ; H^{s}\left(\mathbb{R}^{2}\right)\right)$ holds.

(ii) The size of $|\xi+\eta|$, which comes from the symbol of the dissipative term of (1.6), is not decided by the size of $|(\xi, \eta)|$. Therefore, to use the dissipative effect strictly, we focus on not only $|(\xi, \eta)| \sim N$, but also $|\xi+\eta| \sim M$. This is a different point from $1 D$ case.

(iii) We can assume $\sum_{M \in 2^{\mathbb{Z}}}=\sum_{M \lesssim N}$ since $|\xi+\eta| \lesssim|(\xi, \eta)|$ holds.

We choose $X_{T}^{s, \frac{1}{2}, 1}$ as the solution space. Now, we define the operator $\mathcal{K}$ and $\mathcal{L}$ by

$$
\begin{aligned}
& \mathcal{K} F(t)(\xi, \eta):=\int_{\mathbb{R}} \frac{e^{i t \tau}-e^{-|t|(\xi+\eta)^{2}}}{(\xi+\eta)^{2}+i \tau} \mathcal{F}[U(-\cdot) F(\cdot)](\tau, \xi, \eta) d \tau \\
& \mathcal{L} F(t):=U(t) \int_{\mathbb{R}^{2}} e^{i x \xi} e^{i y \eta} \mathcal{K} F(t)(\xi, \eta) d \xi d \eta=U(t) \mathcal{F}_{x, y}^{-1}[\mathcal{K} F(t)]
\end{aligned}
$$

Then, we note that

$$
\mathcal{L} F(t)=\int_{0}^{t} W\left(t-t^{\prime}\right) F\left(t^{\prime}\right) d t^{\prime}
$$

holds for $t \geq 0$ and the integral form of (1.6) on $[0, \infty)$ is given by

$$
\begin{aligned}
v(t) & =W(t) v_{0}+\int_{0}^{t} W\left(t-t^{\prime}\right)\left(\partial_{x}+\partial_{y}\right)\left(v\left(t^{\prime}\right)^{2}\right) d t^{\prime} \\
& =W(t) v_{0}+\mathcal{L}\left(\left(\partial_{x}+\partial_{y}\right) v\right)(t) .
\end{aligned}
$$

Proposition 2.3. Let $s \in \mathbb{R}$. There exists $C_{1}>0$, such that for any $u_{0} \in$ $H^{s}\left(\mathbb{R}^{2}\right)$, we have

$$
\left\|\psi(t) W(t) u_{0}\right\|_{X^{s, \frac{1}{2}, 1}} \leq C_{1}\left\|u_{0}\right\|_{H^{s}} .
$$

Proof. Since

$$
\left(\sum_{N} \sum_{M}\langle N\rangle^{2 s}\left\|P_{N, M} u_{0}\right\|_{L_{x y}^{2}}^{2}\right)^{\frac{1}{2}} \sim\left\|u_{0}\right\|_{H^{s}}
$$

holds, it suffice to prove

$$
\sum_{L}\left\langle M^{2}+L\right\rangle^{\frac{1}{2}}\left\|P_{N, M} Q_{L}\left(\psi(t) W(t) u_{0}\right)\right\|_{L_{t x y}^{2}} \lesssim\left\|P_{N, M} u_{0}\right\|_{L_{x y}^{2}}
$$

for each $N, M \in 2^{\mathbb{Z}}$. By using Plancherel's theorem, we have

$$
\begin{aligned}
& \left\|P_{N, M} Q_{L}\left(\psi(t) W(t) u_{0}\right)\right\|_{L_{t x y}^{2}} \\
& \sim\left\|\varphi_{N, M}(\xi, \eta) \varphi_{L}(\tau) \mathcal{F}_{t}\left[\psi(t) e^{-|t|(\xi+\eta)^{2}}\right] \widehat{u_{0}}(\xi, \eta)\right\|_{L_{\xi \eta t}^{2}} \\
& \lesssim\left\|P_{N, M} u_{0}\right\|_{L_{x y}^{2}}\left\|\phi_{M}(\xi+\eta) \varphi_{L}(\tau) \mathcal{F}_{t}\left[\psi(t) e^{-|t|(\xi+\eta)^{2}}\right]\right\|_{L_{\xi \eta}^{\infty} L_{t}^{2}} \\
& =\left\|P_{N, M} u_{0}\right\|_{L_{x y}^{2}}\left\|\phi_{M}(\zeta) \varphi_{L}(\tau) \mathcal{F}_{t}\left[\psi(t) e^{-|t| \zeta^{2}}\right]\right\|_{L_{\zeta}^{\infty} L_{t}^{2}},
\end{aligned}
$$

where $\phi_{M}=\varphi_{2 M}+\varphi_{M}+\varphi_{\frac{M}{2}}$ and we used $\varphi_{M}=\varphi_{M} \phi_{M}$. Therefore, it suffice to prove

$$
\sum_{L}\left\langle M^{2}+L\right\rangle^{\frac{1}{2}}\left\|\phi_{M}(\zeta) \varphi_{L}(\tau) \mathcal{F}_{t}\left[\psi(t) e^{-|t| \zeta^{2}}\right]\right\|_{L_{\zeta}^{\infty} L_{\tau}^{2}} \lesssim 1
$$

It is obtained in the proof of Proposition 4.1 in 21 . 
Proposition 2.4. Let $s \in \mathbb{R}$. There exists $C_{2}>0$, such that for any $F \in$ $X^{s,-\frac{1}{2}, 1}$, we have

$$
\|\psi(t) \mathcal{L} F(t)\|_{X^{s, \frac{1}{2}, 1}} \leq C_{2}\|F\|_{X^{s,-\frac{1}{2}, 1}}
$$

Proof. We use the argument in the proof of Lemma 4.1 in [21]. Since

$$
\left\|P_{N, M} Q_{L}(\psi(t) \mathcal{L} F(t))\right\|_{L_{t x y}^{2}} \sim\left\|\varphi_{N, M}(\xi, \eta) \varphi_{L}(\tau) \mathcal{F}_{t}[\psi \mathcal{K} F](\tau, \xi, \eta)\right\|_{L_{\xi \eta \tau}^{2}},
$$

it suffice to show that

$$
\begin{aligned}
& \sum_{L}\left\langle M^{2}+L\right\rangle^{\frac{1}{2}}\left\|\varphi_{N, M}(\xi, \eta) \varphi_{L}(\tau) \mathcal{F}_{t}[\psi \mathcal{K} F](\tau, \xi, \eta)\right\|_{L_{\xi \eta \tau}^{2}} \\
& \lesssim \sum_{L}\left\langle M^{2}+L\right\rangle^{-\frac{1}{2}}\left\|\varphi_{N, M}(\xi, \eta) \varphi_{L}(\tau) \mathcal{F}[U(-\cdot) F(\cdot)](\tau, \xi, \eta)\right\|_{L_{\xi \eta \tau}^{2}}
\end{aligned}
$$

We put $w(t)=U(-t) F(t)$ and split $\psi \mathcal{K} F$ into $K_{1}+K_{2}+K_{3}-K_{4}$, where

$$
\begin{aligned}
& K_{1}(t, \xi, \eta)=\psi(t) \int_{|\tau| \leq 1} \frac{e^{i t \tau}-1}{(\xi+\eta)^{2}+i \tau} \widetilde{w}(\tau, \xi, \eta) d \tau \\
& K_{2}(t, \xi, \eta)=\psi(t) \int_{|\tau| \leq 1} \frac{1-e^{-|t|(\xi+\eta)^{2}}}{(\xi+\eta)^{2}+i \tau} \widetilde{w}(\tau, \xi, \eta) d \tau \\
& K_{3}(t, \xi, \eta)=\psi(t) \int_{|\tau| \geq 1} \frac{e^{i t \tau}}{(\xi+\eta)^{2}+i \tau} \widetilde{w}(\tau, \xi, \eta) d \tau \\
& K_{4}(t, \xi, \eta)=\psi(t) \int_{|\tau| \geq 1} \frac{e^{-|t|(\xi+\eta)^{2}}}{(\xi+\eta)^{2}+i \tau} \widetilde{w}(\tau, \xi, \eta) d \tau
\end{aligned}
$$

Furthermore, we put $w_{N, M}=P_{N, M} w$. We note that $\widetilde{w}_{N, M}(\tau, \xi, \eta)=\phi_{M}(\xi+$ $\eta) \widetilde{w}_{N, M}(\tau, \xi, \eta)$ since $\varphi_{M}=\varphi_{M} \phi_{M}$.

$\underline{\text { Estimate for } K_{1}}$

By using the Taylor expansion, we have

$$
\begin{aligned}
& \left\|\varphi_{N, M}(\xi, \eta) \varphi_{L}(\tau) \mathcal{F}_{t}\left[K_{1}\right](\tau, \xi, \eta)\right\|_{L_{\xi \eta \tau}^{2}} \\
& \lesssim \sum_{n=1}^{\infty} \frac{1}{n !}\left\|\left(\int_{|\tau| \leq 1} \frac{|\tau|^{n}\left|\widetilde{w}_{N, M}(\tau, \xi, \eta)\right|}{(\xi+\eta)^{2}+|\tau|} d \tau\right)\right\| \varphi_{L}(\tau) \mathcal{F}_{t}\left[t^{n} \psi(t)\right](\tau)\left\|_{L_{\tau}^{2}}\right\|_{L_{\xi \eta}^{2}} .
\end{aligned}
$$

By the Cauchy-Schwartz inequality, we obtain

$$
\begin{aligned}
& \int_{|\tau| \leq 1} \frac{|\tau|^{n}\left|\widetilde{w}_{N, M}(\tau, \xi, \eta)\right|}{(\xi+\eta)^{2}+|\tau|} d \tau \\
& \lesssim\left(\int_{|\tau| \leq 1} \frac{|\tau|^{2}\left\langle(\xi+\eta)^{2}+|\tau|\right\rangle}{\left((\xi+\eta)^{2}+|\tau|\right)^{2}}\left|\phi_{M}(\xi+\eta)\right|^{2} d \tau\right)^{\frac{1}{2}}\left(\int_{|\tau| \leq 1} \frac{\left|\widetilde{w}_{N, M}(\tau, \xi, \eta)\right|^{2}}{\left\langle(\xi+\eta)^{2}+|\tau|\right\rangle} d \tau\right)^{\frac{1}{2}} \\
& \lesssim\langle M\rangle^{-1} \sum_{L}\left\langle M^{2}+L\right\rangle^{-\frac{1}{2}}\left\|\varphi_{L}(\tau) \widetilde{w}_{N, M}(\tau, \xi, \eta)\right\|_{L_{\tau}^{2}}
\end{aligned}
$$


for $n \geq 1$. Therefore, we get

$$
\begin{aligned}
& \sum_{L}\left\langle M^{2}+L\right\rangle^{\frac{1}{2}}\left\|\varphi_{N, M}(\xi, \eta) \varphi_{L}(\tau) \mathcal{F}_{t}\left[K_{1}\right](\tau, \xi, \eta)\right\|_{L_{\xi \eta \tau}^{2}} \\
& \lesssim \sum_{n=1}^{\infty} \frac{1}{n !}\left\||t|^{n} \psi\right\|_{B_{2,1}^{\frac{1}{2}}} \sum_{L}\left\langle M^{2}+L\right\rangle^{-\frac{1}{2}}\left\|\varphi_{L}(\tau) \widetilde{w}_{N, M}(\tau, \xi, \eta)\right\|_{L_{\xi \eta \tau}^{2}} \\
& \lesssim \sum_{L}\left\langle M^{2}+L\right\rangle^{-\frac{1}{2}}\left\|\varphi_{L}(\tau) \widetilde{w}_{N, M}(\tau, \xi, \eta)\right\|_{L_{\xi \eta \tau}^{2}}
\end{aligned}
$$

since $\left\langle M^{2}+L\right\rangle^{\frac{1}{2}}\langle M\rangle^{-1} \lesssim\langle L\rangle^{\frac{1}{2}}$.

\section{Estimate for $K_{2}$}

By Plancherel's theorem, we have

$\left\|\varphi_{N, M}(\xi, \eta) \varphi_{L}(\tau) \mathcal{F}_{t}\left[K_{2}\right](\tau, \xi, \eta)\right\|_{L_{\xi \eta \tau}^{2}}$

$\lesssim\left\|\left(\int_{|\tau| \leq 1} \frac{\left|\widetilde{w}_{N, M}(\tau, \xi, \eta)\right|}{(\xi+\eta)^{2}+|\tau|} d \tau\right)\right\| \phi_{M}(\xi+\eta) \varphi_{L}(\tau) \mathcal{F}_{t}\left[\psi(t)\left(1-e^{-|t|(\xi+\eta)^{2}}\right)\right](\tau)\left\|_{L_{\tau}^{2}}\right\|_{L_{\xi \eta}^{2}}$.

By the Cauchy-Schwartz inequality, we obtain

$$
\begin{aligned}
& \int_{|\tau| \leq 1} \frac{\left|\widetilde{w}_{N, M}(\tau, \xi, \eta)\right|}{(\xi+\eta)^{2}+|\tau|} d \tau \\
& \lesssim\left(\int_{|\tau| \leq 1} \frac{\left\langle(\xi+\eta)^{2}+|\tau|\right\rangle}{\left((\xi+\eta)^{2}+|\tau|\right)^{2}}\left|\phi_{M}(\xi+\eta)\right|^{2} d \tau\right)^{\frac{1}{2}}\left(\int_{|\tau| \leq 1} \frac{\left|\widetilde{w}_{N, M}(\tau, \xi, \eta)\right|^{2}}{\left\langle(\xi+\eta)^{2}+|\tau|\right\rangle} d \tau\right)^{\frac{1}{2}} \\
& \lesssim M^{-2}\langle M\rangle \sum_{L}\left\langle M^{2}+L\right\rangle^{-\frac{1}{2}}\left\|\varphi_{L}(\tau) \widetilde{w}_{N, M}(\tau, \xi, \eta)\right\|_{L_{\tau}^{2}}
\end{aligned}
$$

Therefore if $M \geq 1$, then we get

$$
\begin{aligned}
& \sum_{L}\left\langle M^{2}+L\right\rangle^{\frac{1}{2}}\left\|\varphi_{N, M}(\xi, \eta) \varphi_{L}(\tau) \mathcal{F}_{t}\left[K_{2}\right](\tau, \xi, \eta)\right\|_{L_{\xi \eta \tau}^{2}} \\
& \lesssim \sum_{L}\left\langle M^{2}+L\right\rangle^{-\frac{1}{2}}\left\|\varphi_{L}(\tau) \widetilde{w}_{N, M}(\tau, \xi, \eta)\right\|_{L_{\xi \eta \tau}^{2}}
\end{aligned}
$$

by (2.2) and

$$
\sum_{L}\left\langle M^{2}+L\right\rangle^{\frac{1}{2}}\left\|\varphi_{L}(\tau) \mathcal{F}_{t}[\psi](\tau)\right\|_{L_{\tau}^{2}} \lesssim M\|\psi\|_{B_{2,1}^{\frac{1}{2}}} \lesssim M
$$

While if $M \leq 1$, then by using the Taylor expansion, we have

$$
\begin{aligned}
& \left\|\phi_{M}(\xi+\eta) \varphi_{L}(\tau) \mathcal{F}_{t}\left[\psi(t)\left(1-e^{-|t|(\xi+\eta)^{2}}\right)\right](\tau)\right\|_{L_{\tau}^{2}} \\
& \lesssim \sum_{n=1}^{\infty} \frac{(\xi+\eta)^{2 n}}{n !} \phi_{M}(\xi+\eta)\left\|\varphi_{L}(\tau) \mathcal{F}_{t}\left[\psi(t)|t|^{n}\right](\tau)\right\|_{L_{\tau}^{2}} \\
& \lesssim M^{2} \sum_{n=1}^{\infty} \frac{1}{n !}\left\|\varphi_{L}(\tau) \mathcal{F}_{t}\left[\psi(t)|t|^{n}\right](\tau)\right\|_{L_{\tau}^{2}}
\end{aligned}
$$


Therefore, we get

$$
\begin{aligned}
& \sum_{L}\left\langle M^{2}+L\right\rangle^{\frac{1}{2}}\left\|\varphi_{N, M}(\xi, \eta) \varphi_{L}(\tau) \mathcal{F}_{t}\left[K_{2}\right](\tau, \xi, \eta)\right\|_{L_{\xi \eta \tau}^{2}} \\
& \lesssim \sum_{n=1}^{\infty} \frac{1}{n !}\left\||t|^{n} \psi\right\|_{B_{2,1}^{\frac{1}{2}}} \sum_{L}\left\langle M^{2}+L\right\rangle^{-\frac{1}{2}}\left\|\varphi_{L}(\tau) \widetilde{w}_{N, M}(\tau, \xi, \eta)\right\|_{L_{\xi \eta \tau}^{2}} \\
& \lesssim \sum_{L}\left\langle M^{2}+L\right\rangle^{-\frac{1}{2}}\left\|\varphi_{L}(\tau) \widetilde{w}_{N, M}(\tau, \xi, \eta)\right\|_{L_{\xi \eta \tau}^{2}} .
\end{aligned}
$$

Estimate for $K_{3}$

We put $g_{N, M}(t)=\mathcal{F}_{t}^{-1}\left[\mathbf{1}_{|\tau| \geq 1}\left((\xi+\eta)^{2}+i \tau\right)^{-1} \widetilde{w}_{N, M}(\tau, \xi, \eta)\right](t)$. Then, we have

$$
\begin{aligned}
\left|\varphi_{N, M}(\xi, \eta) \varphi_{L}(\tau) \mathcal{F}_{t}\left[K_{3}\right](\tau)\right| & \sim\left|\varphi_{L}(\tau)\left(\mathcal{F}_{t}[\psi] *_{\tau} \mathcal{F}_{t}\left[g_{N, M}\right](\tau)\right)\right| \\
& \lesssim \sum_{L_{1}} \sum_{L_{2}} \mid \varphi_{L}(\tau)\left(\varphi_{L_{1}} \mathcal{F}_{t}[\psi]\right) *_{\tau}\left(\varphi_{L_{2}} \mathcal{F}_{t}\left[g_{N, M}\right)(\tau) \mid\right.
\end{aligned}
$$

(i) Summation for $L_{1} \ll L$ (then, $L_{2} \sim L$.)

By the Young inequality, we have

$$
\begin{aligned}
& \| \varphi_{L}(\tau)\left(\varphi_{L_{1}} \mathcal{F}_{t}[\psi]\right) *_{\tau}\left(\varphi_{L_{2}} \mathcal{F}_{t}\left[g_{N, M}\right)(\tau) \|_{L_{\tau}^{2}}\right. \\
& \lesssim\left\|\varphi_{L_{1}}(\tau) \mathcal{F}_{t}[\psi](\tau)\right\|_{L_{\tau}^{1}}\left\|\varphi_{L_{2}}(\tau) \mathcal{F}_{t}\left[g_{N, M}\right](\tau)\right\|_{L_{\tau}^{2}} \\
& \lesssim\left\|\varphi_{L_{1}}(\tau) \mathcal{F}_{t}[\psi](\tau)\right\|_{L_{\tau}^{1}}\left\langle M^{2}+L_{2}\right\rangle^{-1}\left\|\varphi_{L_{2}}(\tau) \widetilde{w}_{N, M}(\tau)\right\|_{L_{\tau}^{2}} .
\end{aligned}
$$

Therefore, we obtain

$$
\begin{aligned}
& \sum_{L}\left\langle M^{2}+L\right\rangle^{\frac{1}{2}} \sum_{L_{1} \ll L} \sum_{L_{2} \sim L}\left\|\varphi_{L}(\tau)\left(\varphi_{L_{1}} \mathcal{F}_{t}[\psi]\right) *_{\tau}\left(\varphi_{L_{2}} \mathcal{F}_{t}\left[g_{N, M}\right]\right)(\tau)\right\|_{L_{\xi \eta \tau}^{2}} \\
& \lesssim\left(\sum_{L_{1}}\left\|\varphi_{L_{1}}(\tau) \mathcal{F}_{t}[\psi](\tau)\right\|_{L_{\tau}^{1}}\right)\left(\sum_{L_{2}}\left\langle M^{2}+L_{2}\right\rangle^{-\frac{1}{2}}\left\|\varphi_{L_{2}}(\tau) \widetilde{w}_{N, M}(\tau, \xi, \eta)\right\|_{L_{\xi \eta \tau}^{2}}\right) \\
& \lesssim \sum_{L_{2}}\left\langle M^{2}+L_{2}\right\rangle^{-\frac{1}{2}}\left\|\varphi_{L_{2}}(\tau) \widetilde{w}_{N, M}(\tau, \xi, \eta)\right\|_{L_{\xi \eta \tau}^{2}}
\end{aligned}
$$

since

$$
\sum_{L_{1}}\left\|\varphi_{L_{1}}(\tau) \mathcal{F}_{t}[\psi](\tau)\right\|_{L_{\tau}^{1}} \lesssim \sum_{L_{1}} L_{1}^{\frac{1}{2}}\left\|\varphi_{L_{1}}(\tau) \mathcal{F}_{t}[\psi](\tau)\right\|_{L_{\tau}^{2}} \lesssim\|\psi\|_{B_{2,1}^{\frac{1}{2}}} \lesssim 1
$$

(ii) Summation for $L \lesssim M^{2}, L_{1} \gtrsim L$.

By the Hölder inequality and the Young inequality, we have

$$
\begin{aligned}
& \left\|\varphi_{L}(\tau)\left(\varphi_{L_{1}} \mathcal{F}_{t}[\psi]\right) *_{\tau}\left(\varphi_{L_{2}} \mathcal{F}_{t}\left[g_{N, M}\right]\right)(\tau)\right\|_{L_{\tau}^{2}} \\
& \lesssim\left\|\varphi_{L}\right\|_{L_{\tau}^{2}}\left\|\varphi_{L_{1}}(\tau) \mathcal{F}_{t}[\psi](\tau)\right\|_{L_{\tau}^{2}}\left\|\varphi_{L_{2}}(\tau) \mathcal{F}_{t}\left[g_{N, M}\right](\tau)\right\|_{L_{\tau}^{2}} \\
& \lesssim L^{\frac{1}{2}}\left\|\varphi_{L_{1}}(\tau) \mathcal{F}_{t}[\psi](\tau)\right\|_{L_{\tau}^{2}}\left\langle M^{2}+L_{2}\right\rangle^{-1}\left\|\varphi_{L_{2}}(\tau) \widetilde{w}_{N, M}(\tau)\right\|_{L_{\tau}^{2}} .
\end{aligned}
$$


Therefore, we obtain

$$
\begin{aligned}
& \sum_{L \lesssim M^{2}}\left\langle M^{2}+L\right\rangle^{\frac{1}{2}} \sum_{L_{1} \gtrsim L} \sum_{L_{2}}\left\|\varphi_{L}(\tau)\left(\varphi_{L_{1}} \mathcal{F}_{t}[\psi]\right) *_{\tau}\left(\varphi_{L_{2}} \mathcal{F}_{t}\left[g_{N, M}\right]\right)(\tau)\right\|_{L_{\xi \eta \tau}^{2}} \\
& \lesssim\langle M\rangle\left(\sum_{L_{1}} L_{1}^{\frac{1}{2}}\left\|\varphi_{L_{1}}(\tau) \mathcal{F}_{t}[\psi](\tau)\right\|_{L_{\tau}^{2}}\right)\left(\sum_{L_{2}}\left\langle M^{2}+L_{2}\right\rangle^{-1}\left\|\varphi_{L_{2}}(\tau) \widetilde{w}_{N, M}(\tau, \xi, \eta)\right\|_{L_{\xi \eta \tau}^{2}}\right) \\
& \lesssim \sum_{L_{2}}\left\langle M^{2}+L_{2}\right\rangle^{-\frac{1}{2}}\left\|\varphi_{L_{2}}(\tau) \widetilde{w}_{N, M}(\tau, \xi, \eta)\right\|_{L_{\xi \eta \tau}^{2}}
\end{aligned}
$$

since $\langle M\rangle \lesssim\left\langle M^{2}+L_{2}\right\rangle^{\frac{1}{2}}$ and

$$
\sum_{L_{1}} L_{1}^{\frac{1}{2}}\left\|\varphi_{L_{1}}(\tau) \mathcal{F}_{t}[\psi](\tau)\right\|_{L_{\tau}^{2}} \lesssim\|\psi\|_{B_{2,1}^{\frac{1}{2}}} \lesssim 1
$$

(iii) Summation for $L_{1} \gtrsim L \gtrsim M^{2}$. By the Young inequality and the CauchySchwartz inequality, we have

$$
\begin{aligned}
& \left\|\varphi_{L}(\tau)\left(\varphi_{L_{1}} \mathcal{F}_{t}[\psi]\right) *_{\tau}\left(\varphi_{L_{2}} \mathcal{F}_{t}\left[g_{N, M}\right]\right)(\tau)\right\|_{L_{\tau}^{2}} \\
& \lesssim\left\|\varphi_{L_{1}}(\tau) \mathcal{F}_{t}[\psi](\tau)\right\|_{L_{\tau}^{2}}\left\|\varphi_{L_{2}}(\tau) \mathcal{F}_{t}\left[g_{N, M}\right](\tau)\right\|_{L_{\tau}^{1}} \\
& \lesssim\left\|\varphi_{L_{1}}(\tau) \mathcal{F}_{t}[\psi](\tau)\right\|_{L_{\tau}^{2}}\left\langle M^{2}+L_{2}\right\rangle^{-\frac{1}{2}}\left\|\varphi_{L_{2}}(\tau) \widetilde{w}_{N, M}(\tau)\right\|_{L_{\tau}^{2}} .
\end{aligned}
$$

Therefore, we obtain

$$
\begin{aligned}
& \sum_{L \gtrsim M^{2}}\left\langle M^{2}+L\right\rangle^{\frac{1}{2}} \sum_{L_{1} \gtrsim L} \sum_{L_{2}}\left\|\varphi_{L}(\tau)\left(\varphi_{L_{1}} \mathcal{F}_{t}[\psi]\right) *_{\tau}\left(\varphi_{L_{2}} \mathcal{F}_{t}\left[g_{N, M}\right]\right)(\tau)\right\|_{L_{\xi \eta \tau}^{2}} \\
& \lesssim\left(\sum_{L_{1}}\left\langle L_{1}\right\rangle^{\frac{1}{2}}\left\|\varphi_{L_{1}}(\tau) \mathcal{F}_{t}[\psi](\tau)\right\|_{L_{\tau}^{2}}\right)\left(\sum_{L_{2}}\left\langle M^{2}+L_{2}\right\rangle^{-\frac{1}{2}}\left\|\varphi_{L_{2}}(\tau) \widetilde{w}_{N, M}(\tau, \xi, \eta)\right\|_{L_{\xi \eta \tau}^{2}}\right) \\
& \lesssim \sum_{L_{2}}\left\langle M^{2}+L_{2}\right\rangle^{-\frac{1}{2}}\left\|\varphi_{L_{2}}(\tau) \widetilde{w}_{N, M}(\tau, \xi, \eta)\right\|_{L_{\xi \eta \tau}^{2}}
\end{aligned}
$$

since

$$
\sum_{L_{1}}\left\langle L_{1}\right\rangle^{\frac{1}{2}}\left\|\varphi_{L_{1}}(\tau) \mathcal{F}_{t}[\psi](\tau)\right\|_{L_{\tau}^{2}} \lesssim\|\psi\|_{B_{2,1}^{\frac{1}{2}}} \lesssim 1
$$

Estimate for $K_{4}$

By Plancherel's theorem, we have

$\left\|\varphi_{N, M}(\xi, \eta) \varphi_{L}(\tau) \mathcal{F}_{t}\left[K_{4}\right](\tau, \xi, \eta)\right\|_{L_{\xi \eta \tau}^{2}}$

$\lesssim\left\|\left(\int_{|\tau| \geq 1} \frac{\left|\widetilde{w}_{N, M}(\tau, \xi, \eta)\right|}{(\xi+\eta)^{2}+|\tau|} d \tau\right)\right\| \phi_{M}(\xi+\eta) \varphi_{L}(\tau) \mathcal{F}_{t}\left[\psi(t) e^{-|t|(\xi+\eta)^{2}}\right](\tau)\left\|_{L_{\tau}^{2}}\right\|_{L_{\xi \eta}^{2}}$.

By the Cauchy-Schwartz inequality, we obtain

$$
\begin{aligned}
\int_{|\tau| \geq 1} \frac{\left|\widetilde{w}_{N, M}(\tau, \xi, \eta)\right|}{(\xi+\eta)^{2}+|\tau|} d \tau & \lesssim \sum_{L}\left\langle M^{2}+L\right\rangle^{-1}\left\|\varphi_{L}(\tau) \widetilde{w}_{N, M}(\tau, \xi, \eta)\right\|_{L_{\tau}^{1}} \\
& \lesssim \sum_{L}\left\langle M^{2}+L\right\rangle^{-\frac{1}{2}}\left\|\varphi_{L}(\tau) \widetilde{w}_{N, M}(\tau, \xi, \eta)\right\|_{L_{\tau}^{2}}
\end{aligned}
$$


Therefore, by (2.2), we get

$$
\begin{aligned}
& \sum_{L}\left\langle M^{2}+L\right\rangle^{\frac{1}{2}}\left\|\varphi_{N, M}(\xi, \eta) \varphi_{L}(\tau) \mathcal{F}_{t}\left[K_{4}\right](\tau, \xi, \eta)\right\|_{L_{\xi \eta \tau}^{2}} \\
& \lesssim \sum_{L}\left\langle M^{2}+L\right\rangle^{-\frac{1}{2}}\left\|\varphi_{L}(\tau) \widetilde{w}_{N, M}(\tau, \xi, \eta)\right\|_{L_{\xi \eta \tau}^{2}} .
\end{aligned}
$$

\section{Bilinear estimate}

In this section, we prove the estimate for nonlinear term as follows.

Proposition 3.1. Let $s \geq s_{0}>-\frac{1}{2}$. There exist $0<\delta \ll 1$ and $C_{3}>0$, such that for any $u, v \in X^{s, \frac{1-\delta}{2}, 1}$, we have

$$
\left\|\left(\partial_{x}+\partial_{y}\right)(u v)\right\|_{X^{s,-\frac{1}{2}, 1}} \leq C_{3}\|u\|_{X^{s, \frac{1-\delta}{2}, 1}}\|v\|_{X^{s, \frac{1-\delta}{2}, 1}} .
$$

To prove Proposition 3.1, we first give some Strichartz estimates.

Proposition 3.2. Let $(p, q) \in \mathbb{R}^{2}$ satisfy $p \geq 3$ and $\frac{3}{p}+\frac{2}{q}=1$. For any $u_{0} \in L^{2}\left(\mathbb{R}^{2}\right)$, we have

$$
\left\|U(t) u_{0}\right\|_{L_{t}^{p} L_{x y}^{q}} \lesssim\left\|u_{0}\right\|_{L_{x y}^{2}} .
$$

Proposition 3.2 is obtained by using the variable transform $(x, y) \mapsto\left(4^{-\frac{1}{3}}(x+\right.$ $\left.\sqrt{3} y), 4^{-\frac{1}{3}}(x-\sqrt{3} y)\right)$ in Proposition 2.4 in [17.

Proposition 3.3. For any $u_{0} \in L^{2}\left(\mathbb{R}^{2}\right)$, we have

$$
\left\|D_{x}^{\frac{1}{8}} D_{y}^{\frac{1}{8}} U(t) u_{0}\right\|_{L_{t x y}^{4}} \lesssim\left\|u_{0}\right\|_{L_{x y}^{2}},
$$

where $D_{x}^{s}=\mathcal{F}_{x y}^{-1}|\xi|^{s} \mathcal{F}_{x y}, D_{y}^{s}=\mathcal{F}_{x y}^{-1}|\eta|^{s} \mathcal{F}_{x y}$ for $s \in \mathbb{R}$.

Proposition 3.3 is obtained by applying $\Omega(\xi, \eta)=\xi^{3}+\eta^{3}$ in Corollary 3.4 in [19.

By using the same argument as in Lemma 2.3 in $[8$, we obtain the following estimates from Proposition 3.2 and Proposition 3.3 .

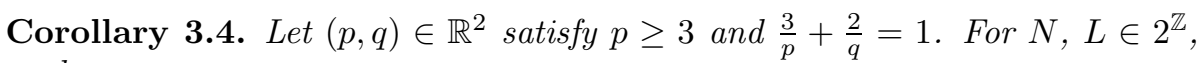
we have

$$
\left\|P_{N} Q_{L} u\right\|_{L_{t}^{p} L_{x y}^{q}} \lesssim L^{\frac{1}{2}}\left\|P_{N} Q_{L} u\right\|_{L_{t x y}^{2}} .
$$

Furthermore, if $\mathcal{F}_{x y}\left[P_{N} u\right]$ is supported in $\{(\xi, \eta)|| \xi|\sim| \eta \mid\}$, then we have

$$
\left\|P_{N} Q_{L} u\right\|_{L_{t x y}^{4}} \lesssim N^{-\frac{1}{4}} L^{\frac{1}{2}}\left\|P_{N} Q_{L} u\right\|_{L_{t x y}^{2}} .
$$

To get a positive power of $M$, we give the following estimates.

Corollary 3.5. Let $0<\delta \ll 1,0<\epsilon<1-\delta$. For $N, M, L \in 2^{\mathbb{Z}}$, we have

$$
\left\|P_{N, M} Q_{L} u\right\|_{L_{t x y}^{\frac{4}{1+\delta}}} \lesssim(N M)^{\frac{\epsilon}{4}} L^{\frac{5(1-\delta)}{12}-\frac{\epsilon}{6}}\left\|P_{N, M} Q_{L} u\right\|_{L_{t x y}^{2}} .
$$

Furthermore, if $\mathcal{F}_{x y}\left[P_{N} u\right]$ is supported in $\{(\xi, \eta)|| \xi|\sim| \eta \mid\}$, then we have

$$
\left\|P_{N, M} Q_{L} u\right\|_{L_{t x y}^{\frac{4}{1+\delta}}} \lesssim(N M)^{\frac{\epsilon}{4}} N^{-\frac{1}{4}(1-\delta-\epsilon)} L^{\frac{1-\delta}{2}-\frac{\epsilon}{4}}\left\|P_{N} Q_{L} u\right\|_{L_{t x y}^{2}} .
$$


Proof. By (3.1) with $p=q=5$, we have the $L^{5}$-Strichartz estimate

$$
\left\|P_{N, M} Q_{L} u\right\|_{L_{t x y}^{5}} \lesssim L^{\frac{1}{2}}\left\|P_{N, M} Q_{L} u\right\|_{L_{t x y}^{2}} .
$$

By the interpolation between (3.5) and a trivial equality $\left\|P_{N, M} Q_{L} u\right\|_{L_{t x y}^{2}}=$ $L^{0}\left\|P_{N, M} Q_{L} u\right\|_{L_{t x y}^{2}}$, we have

$$
\left\|P_{N, M} Q_{L} u\right\|_{L_{t x y}^{\frac{4-2 \epsilon}{1+\delta}}} \lesssim L^{\frac{5(1-\delta-\epsilon)}{6(2-\epsilon)}}\left\|P_{N} Q_{L} u\right\|_{L_{t x y}^{2}} .
$$

While, by the Cauchy-Schwartz inequality, we obtain

$$
\begin{aligned}
\left\|P_{N, M} Q_{L} u\right\|_{L_{x y}^{\infty}} & \leq \int_{\substack{|(\xi, \eta)| \sim N \\
|\xi+\eta| \sim M}}\left|\mathcal{F}_{x y}\left[P_{N, M} Q_{L} u\right](\xi, \eta)\right| d \xi d \eta \\
& \lesssim(N M)^{\frac{1}{2}}\left\|P_{N, M} Q_{L} u\right\|_{L_{x y}^{2}} .
\end{aligned}
$$

Therefore, by using (3.1) with $(p, q)=(\infty, 2)$, we have

$$
\left\|P_{N, M} Q_{L} u\right\|_{L_{t x y}^{\infty}} \lesssim(N M L)^{\frac{1}{2}}\left\|P_{N, M} Q_{L} u\right\|_{L_{t x y}^{2}} .
$$

By the interpolation between (3.6) and (3.7), we obtain (3.3).

By using (3.2) instead of (3.5) in the above argument, we also get (3.4).

Next, we give the bilinear Strichartz estimates.

Proposition 3.6. Let $R_{K}^{(j)}(j=1,2)$ denote the bilinear operator defined by $\mathcal{F}_{x y}\left[R_{K}^{(1)}\left(u_{1}, u_{2}\right)\right](\xi, \eta)=\int \varphi_{K}\left(\xi_{1}^{2}-\left(\xi-\xi_{1}\right)^{2}\right) \widehat{u_{1}}\left(\xi_{1}, \eta_{1}\right) \widehat{u_{2}}\left(\xi-\xi_{1}, \eta-\eta_{1}\right) d \xi_{1} d \eta_{1}$, $\mathcal{F}_{x y}\left[R_{K}^{(2)}\left(u_{1}, u_{2}\right)\right](\xi, \eta)=\int \varphi_{K}\left(\eta_{1}^{2}-\left(\eta-\eta_{1}\right)^{2}\right) \widehat{u_{1}}\left(\xi_{1}, \eta_{1}\right) \widehat{u_{2}}\left(\xi-\xi_{1}, \eta-\eta_{1}\right) d \xi_{1} d \eta_{1}$.

For $N_{1}, N_{2}, L_{1}, L_{2}, K \in 2^{\mathbb{Z}}$ with $N_{1} \geq N_{2}$, and $j \in\{1,2\}$, we have

$$
\begin{aligned}
& \left\|R_{K}^{(j)}\left(P_{N_{1}} Q_{L_{1}} u_{1}, P_{N_{2}} Q_{L_{2}} u_{2}\right)\right\|_{L_{t x y}^{2}} \\
& \lesssim K^{-\frac{1}{2}} N_{2}^{\frac{1}{2}} L_{1}^{\frac{1}{2}} L_{2}^{\frac{1}{2}}\left\|P_{N_{1}} Q_{L_{1}} u_{1}\right\|_{L_{t x y}^{2}}^{2}\left\|P_{N_{2}} Q_{L_{2}} u_{2}\right\|_{L_{t x y}^{2}} .
\end{aligned}
$$

Proof. We only prove for $j=1$ because the case $j=2$ can be proved by the same way. We put $f_{i}=\mathcal{F}\left[P_{N_{i}} Q_{L_{i}} u_{i}\right], \zeta_{i}=\left(\xi_{i}, \eta_{i}\right)(i=1,2)$. By the duality argument, it suffice to show that

$$
\begin{aligned}
& \left|\int_{\Omega} f_{1}\left(\tau_{1}, \zeta_{1}\right) f_{2}\left(\tau_{2}, \zeta_{2}\right) f\left(\tau_{1}+\tau_{2}, \zeta_{1}+\zeta_{2}\right) d \tau_{1} d \tau_{2} d \zeta_{1} d \zeta_{2}\right| \\
& \lesssim K^{-\frac{1}{2}} N_{2}^{\frac{1}{2}} L_{1}^{\frac{1}{2}} L_{2}^{\frac{1}{2}}\left\|f_{1}\right\|_{L_{\tau \xi \eta}^{2}}\left\|f_{2}\right\|_{L_{\tau \xi \eta}^{2}}\|f\|_{L_{\tau \xi \eta}^{2}}
\end{aligned}
$$

for any $f \in L^{2}\left(\mathbb{R} \times \mathbb{R}^{2}\right)$, where

$$
\Omega=\left\{\left(\tau_{1}, \tau_{2}, \zeta_{1}, \zeta_{2}\right)|| \zeta_{i}\left|\sim N_{i},\right| \tau_{i}-\xi_{i}^{3}-\eta_{i}^{3}\left|\sim L_{i}(i=1,2),\right| \xi_{1}^{2}-\xi_{2}^{2} \mid \sim K\right\} .
$$


By the Cauchy-Schwartz inequality, we have

$$
\begin{aligned}
& \left|\int_{\Omega} f_{1}\left(\tau_{1}, \zeta_{1}\right) f_{2}\left(\tau_{2}, \zeta_{2}\right) f\left(\tau_{1}+\tau_{2}, \zeta_{1}+\zeta_{2}\right) d \tau_{1} d \tau_{2} d \zeta_{1} d \zeta_{2}\right| \\
& \lesssim\left\|f_{1}\right\|_{L_{\tau \xi \eta}^{2}}\left\|f_{2}\right\|_{L_{\tau \xi \eta}^{2}}\left(\int_{\Omega}\left|f\left(\tau_{1}+\tau_{2}, \zeta_{1}+\zeta_{2}\right)\right|^{2} d \tau_{1} d \tau_{2} d \zeta_{1} d \zeta_{2}\right)^{\frac{1}{2}}
\end{aligned}
$$

By applying the variable transform $\left(\tau_{1}, \tau_{2}\right) \mapsto\left(\theta_{1}, \theta_{2}\right)$ and $\left(\zeta_{1}, \zeta_{2}\right) \mapsto(\mu, w, z, \nu)$ as

$$
\begin{aligned}
& \theta_{i}=\tau_{i}-\xi_{i}^{3}-\eta_{i}^{3}(i=1,2), \\
& \mu=\theta_{1}+\theta_{2}+\xi_{1}^{3}+\xi_{2}^{3}+\eta_{1}^{3}+\eta_{2}^{3}, w=\xi_{1}+\xi_{2}, z=\eta_{1}+\eta_{2}, \nu=\eta_{2},
\end{aligned}
$$

we have

$$
\begin{aligned}
& \int_{\Omega}\left|f\left(\tau_{1}+\tau_{2}, \zeta_{1}+\zeta_{2}\right)\right|^{2} d \tau_{1} d \tau_{2} d \zeta_{1} d \zeta_{2} \\
& \lesssim \int_{\substack{\left|\theta_{1}\right| \sim L_{1} \\
\left|\theta_{2}\right| \sim L_{2}}}\left(\int_{|\nu| \lesssim N_{2}}|f(\mu, w, z)|^{2} \mathbf{1}_{\left\{\left|\xi_{1}^{2}-\xi_{2}^{2}\right| \sim K\right\}}\left(\xi_{1}, \xi_{2}\right) J\left(\zeta_{1}, \zeta_{2}\right)^{-1} d \mu d w d z d \nu\right) d \theta_{1} d \theta_{2}
\end{aligned}
$$

where

$$
J\left(\zeta_{1}, \zeta_{2}\right)=\left|\operatorname{det} \frac{\partial(\mu, w, z, \nu)}{\partial\left(\xi_{1}, \eta_{1}, \xi_{2}, \eta_{2}\right)}\right|=3\left|\xi_{1}^{2}-\xi_{2}^{2}\right| .
$$

Therefore, we obtain

$$
\int_{\Omega}\left|f\left(\tau_{1}+\tau_{2}, \zeta_{1}+\zeta_{2}\right)\right|^{2} d \tau_{1} d \tau_{2} d \zeta_{1} d \zeta_{2} \lesssim K^{-1} N_{2} L_{1} L_{2}\|f\|_{L_{\tau \xi \eta}^{2}}
$$

As a result, we get (3.9) from (3.10) and (3.11).

Remark 3.7. In particullar, if $N_{1} \gg N_{2}$, then we have

$$
\begin{aligned}
& \left\|P_{N_{1}} Q_{L_{1}} u_{1} \cdot P_{N_{2}} Q_{L_{2}} u_{2}\right\|_{L_{t x y}^{2}} \\
& \lesssim N_{1}^{-1} N_{2}^{\frac{1}{2}} L_{1}^{\frac{1}{2}} L_{2}^{\frac{1}{2}}\left\|P_{N_{1}} Q_{L_{1}} u_{1}\right\|_{L_{t x y}^{2}}\left\|P_{N_{2}} Q_{L_{2}} u_{2}\right\|_{L_{t x y}^{2}}
\end{aligned}
$$

since the equality

$$
P_{N_{1}} Q_{L_{1}} u_{1} \cdot P_{N_{2}} Q_{L_{2}} u_{2}=R_{K}^{(j)}\left(P_{N_{1}} Q_{L_{1}} u_{1}, P_{N_{2}} Q_{L_{2}} u_{2}\right)
$$

with $K \sim N_{1}^{2}$ holds for $j=1$ or 2 .

Corollary 3.8. Let $0<\delta \ll 1,0<\epsilon<1-\delta$. For $N_{1}, N_{2}, M_{1}, M_{2}, L_{1}$, $L_{2} \in 2^{\mathbb{Z}}$ with $N_{1} \gg N_{2}$, we have

$$
\begin{aligned}
& \left\|P_{N_{1}, M_{1}} Q_{L_{1}} u_{1} \cdot P_{N_{2}, M_{2}} Q_{L_{2}} u_{2}\right\|_{L_{t x y}^{1+\delta}}^{\frac{2}{1+\delta}} \\
& \lesssim J_{\delta, \epsilon}\left(L_{1} L_{2}\right)^{\frac{1-\delta}{2}-\frac{\epsilon}{4}}\left\|P_{N_{1}, M_{1}} Q_{L_{1}} u_{1}\right\|_{L_{t x y}^{2}}\left\|P_{N_{2}, M_{2}} Q_{L_{2}} u_{2}\right\|_{L_{t x y}^{2}},
\end{aligned}
$$

where

$$
J_{\delta, \epsilon}=J_{\delta, \epsilon}\left(N_{1}, M_{1}, N_{2}, M_{2}\right)=\left(N_{1} M_{1} N_{2} M_{2}\right)^{\frac{\epsilon}{4}}\left(N_{1}^{-1} N_{2}^{\frac{1}{2}}\right)^{1-\delta-\epsilon} .
$$


Proof. By the Hölder inequality and (3.7), we have

$$
\begin{aligned}
& \left\|P_{N_{1}, M_{1}} Q_{L_{1}} u_{1} \cdot P_{N_{2}, M_{2}} Q_{L_{2}} u_{2}\right\|_{L_{t x y}^{2}} \\
& \lesssim\left\|P_{N_{1}, M_{1}} Q_{L_{1}} u_{1}\right\|_{L_{t x y}^{2}}^{\frac{1}{2}}\left\|P_{N_{2}, M_{2}} Q_{L_{2}} u_{2}\right\|_{L_{t x y}^{2}}^{\frac{1}{2}}\left\|P_{N_{1}, M_{1}} Q_{L_{1}} u_{1}\right\|_{L_{t x y}^{2}}^{\frac{1}{2}}\left\|P_{N_{2}, M_{2}} Q_{L_{2}} u_{2}\right\|_{L_{t x y}^{\infty}}^{\frac{1}{2}} \\
& \lesssim\left(N_{1} M_{1} L_{1} N_{2} M_{2} L_{2}\right)^{\frac{1}{4}}\left\|P_{N_{1}, M_{1}} Q_{L_{1}} u_{1}\right\|_{L_{t x y}^{2}}^{2}\left\|P_{N_{2}, M_{2}} Q_{L_{2}} u_{2}\right\|_{L_{t x y}^{2}}^{2} .
\end{aligned}
$$

By the interpolation between this estimate and (3.12), we obtain

$$
\begin{aligned}
& \left\|P_{N_{1}, M_{1}} Q_{L_{1}} u_{1} \cdot P_{N_{2}, M_{2}} Q_{L_{2}} u_{2}\right\|_{L_{t x y}^{2}} \\
& \lesssim J_{\delta, \epsilon}^{\frac{1}{1-\delta}}\left(L_{1} L_{2}\right)^{\frac{1}{2}-\frac{\epsilon}{4(1-\delta)}}\left\|P_{N_{1}, M_{1}} Q_{L_{1}} u_{1}\right\|_{L_{t x y}^{2}}\left\|P_{N_{2}, M_{2}} Q_{L_{2}} u_{2}\right\|_{L_{t x y}^{2}} .
\end{aligned}
$$

While, by the Cauchy-Schwartz inequality, we have

$$
\left\|P_{N_{1}, M_{1}} Q_{L_{1}} u_{1} \cdot P_{N_{2}, M_{2}} Q_{L_{2}} u_{2}\right\|_{L_{t x y}^{1}} \lesssim\left\|P_{N_{1}, M_{1}} Q_{L_{1}} u_{1}\right\|_{L_{t x y}^{2}}\left\|P_{N_{2}, M_{2}} Q_{L_{2}} u_{2}\right\|_{L_{t x y}^{2}} .
$$

By the interpolation between (3.14) and (3.15), we obtain (3.13).

Here, we prove Proposition 3.1 .

Proof of Proposition 3.1. By using the embedding $l^{1} \hookrightarrow l^{2}$ for the summation $\sum_{N} \sum_{M}$, and the duality argument, we have

$$
\begin{aligned}
& \left\|\left(\partial_{x}+\partial_{y}\right)(u v)\right\|_{X^{s,-\frac{1}{2}, 1}} \\
& \lesssim \sum_{N_{1}, M_{1}, L_{1}} \sum_{N_{2}, M_{2}, L_{2}}\left(\sum_{N, M, L} \frac{\langle N\rangle^{s} M}{\left\langle M^{2}+L\right\rangle^{\frac{1}{2}}}\right. \\
& \left.\quad \times \sup _{\|w\|_{L^{2}}=1}\left|\int P_{N_{1}, M_{1}} Q_{L_{1}} u \cdot P_{N_{2}, M_{2}} Q_{L_{2}} v \cdot P_{N, M} Q_{L} w d t d x d y\right|\right) .
\end{aligned}
$$

We put

$$
\begin{aligned}
& \quad u_{N_{1}, M_{1}, L_{1}}=P_{N_{1}, M_{1}} Q_{L_{1}} u, v_{N_{2}, M_{2}, L_{2}}=P_{N_{2}, M_{2}} Q_{L_{2}} v, w_{N, M, L}=P_{N, M} Q_{L} w, \\
& f_{N_{1}, M_{1}, L_{1}}=\left\langle N_{1}\right\rangle^{s}\left\langle M_{1}^{2}+L_{1}\right\rangle^{\frac{1-\delta}{2}} u_{N_{1}, M_{1}, L_{1}}, g_{N_{2}, M_{2}, L_{2}}=\left\langle N_{2}\right\rangle^{s}\left\langle M_{2}^{2}+L_{2}\right\rangle^{\frac{1-\delta}{2}} v_{N_{2}, M_{2}, L_{2}}, \\
& \text { for } 0<\delta \ll 1 \text { and }
\end{aligned}
$$

$$
I=\left|\int u_{N_{1}, M_{1}, L_{1}} \cdot v_{N_{2}, M_{2}, L_{2}} \cdot w_{N, M, L} d t d x d y\right|
$$

We note that $L_{1}^{b}\left\|u_{N_{1}, M_{1}, L_{1}}\right\|_{L_{t x y}^{2}} \lesssim\left\langle N_{1}\right\rangle^{-s}\left\|f_{N_{1}, M_{1}, L_{1}}\right\|_{L_{t x y}^{2}}$ and $L_{2}^{b}\left\|v_{N_{2}, M_{2}, L_{2}}\right\|_{L_{t x y}^{2}} \lesssim$ $\left\langle N_{2}\right\rangle^{-s}\left\|g_{N_{2}, M_{2}, L_{2}}\right\|_{L_{t x y}^{2}}$ hold for $b \leq \frac{1-\delta}{2}$ since $L_{i} \lesssim\left\langle M_{i}^{2}+L_{i}\right\rangle(i=1,2)$.

By the symmetry, we can assume $N_{1} \gtrsim N_{2}$. We first consider the case $1 \geq N_{1} \gtrsim N_{2}$. We note that

$$
\left\|P_{N, M} Q_{L} u\right\|_{L_{t x y}^{\frac{2}{1-\delta}}} \lesssim L^{\frac{5}{6} \delta}\left\|P_{N, M} Q_{L} u\right\|_{L_{t x y}^{2}}
$$


holds by the interpolation between (3.5) and a trivial equality $\left\|P_{N, M} Q_{L} u\right\|_{L_{t x y}^{2}}=$ $L^{0}\left\|P_{N, M} Q_{L} u\right\|_{L_{t x y}^{2}}$. By the Hölder inequality, (3.3), and (3.16), we have

$$
\begin{aligned}
I & \lesssim\left\|u_{N_{1}, M_{1}, L_{1}}\right\|_{L_{t x y}^{\frac{4}{1+\delta}}}\left\|v_{N_{2}, M_{2}, L_{2}}\right\|_{L_{t x y}} \frac{4}{1+\delta}\left\|w_{N, M, L}\right\|_{L_{t x y}^{\frac{2}{1-\delta}}} \\
& \lesssim\left(N_{1} M_{1} N_{2} M_{2}\right)^{\frac{\epsilon}{2}} L^{\frac{5}{6} \delta}\left\|f_{N_{1}, M_{1}, L_{1}}\right\|_{L_{t x y}^{2}}\left\|g_{N_{2}, M_{2}, L_{2}}\right\|_{L_{t x y}^{2}}\left\|w_{N, M, L}\right\|_{L_{t x y}^{2}}
\end{aligned}
$$

since $\left\langle N_{i}\right\rangle^{s} \sim 1(i=1,2)$ for any $s \in \mathbb{R}$. Therefore, we obtain

$$
\begin{aligned}
& \sum_{N \lesssim 1} \sum_{M \lesssim N} \sum_{L} \frac{\langle N\rangle^{s} M}{\left\langle M^{2}+L\right\rangle^{\frac{1}{2}}} \sup _{\|w\|_{L^{2}}=1} I \\
& \lesssim\left(N_{1} M_{1} N_{2} M_{2}\right)^{\frac{\epsilon}{2}}\left\|f_{N_{1}, M_{1}, L_{1}}\right\|_{L_{t x y}^{2}}\left\|g_{N_{2}, M_{2}, L_{2}}\right\|_{L_{t x y}^{2}}
\end{aligned}
$$

since

$$
\sum_{L} \frac{L^{\frac{5}{6} \delta}}{\left\langle M^{2}+L\right\rangle^{\frac{1}{2}}} \lesssim \sum_{L \lesssim\langle M\rangle^{2}} \frac{L^{\frac{5}{6} \delta}}{\langle M\rangle}+\sum_{L \gtrsim\langle M\rangle^{2}} L^{-\left(\frac{1}{2}-\frac{5}{6} \delta\right)} \lesssim\langle M\rangle^{-\left(1-\frac{5}{3} \delta\right)} \lesssim 1
$$

and

$$
\sum_{N \lesssim 1} \sum_{M \lesssim N}\langle N\rangle^{s} M \sim \sum_{N \lesssim 1} \sum_{M \lesssim N} M \lesssim \sum_{N \lesssim 1} N \lesssim 1
$$

for any $s \in \mathbb{R}$. By using (3.17) and the Cauchy-Schwartz inequality for the summations $\sum_{N_{1}, M_{1} \lesssim 1}$ and $\sum_{N_{2}, M_{2} \lesssim 1}$, we have

$$
\sum_{N_{1}, M_{1} \lesssim 1} \sum_{L_{1}} \sum_{N_{2}, M_{2} \lesssim 1} \sum_{L_{2}}\left(\sum_{N, M, L} \frac{\langle N\rangle^{s} M}{\left\langle M^{2}+L\right\rangle^{\frac{1}{2}}} \sup _{\|w\|_{L^{2}=1}} I\right) \lesssim\|u\|_{X^{s, \frac{1-\delta}{2}, 1}}\|v\|_{X^{s, \frac{1-\delta}{2}, 1}}
$$

for any $s \in \mathbb{R}$.

Next, we consider the case $N_{1} \gtrsim N_{2}, N_{1} \geq 1$. It suffice to show that

$$
\begin{aligned}
& \sum_{N, M, L} \frac{\langle N\rangle^{s} M}{\left\langle M^{2}+L\right\rangle^{\frac{1}{2}}} \sup _{\|w\|_{L^{2}}=1} I \\
& \lesssim N_{1}^{-\epsilon}\left(M_{1} M_{2}\right)^{\frac{\epsilon}{4}}\left\|f_{N_{1}, M_{1}, L_{1}}\right\|_{L_{t x y}^{2}}\left\|g_{N_{2}, M_{2}, L_{2}}\right\|_{L_{t x y}^{2}}
\end{aligned}
$$

for small $\epsilon>0$. Indeed, (3.18) and the Cauchy-Schwartz inequality for the summations $\sum_{N_{1}, M_{1}}$ and $\sum_{N_{2}, M_{2}}$ imply

$$
\begin{aligned}
& \sum_{\substack{N_{1}, M_{1}, L_{1} \\
N_{1} \geq 1}} \sum_{\substack{N_{2}, M_{2}, L_{2} \\
N_{2} \lesssim N_{1}}}\left(\sum_{N, M, L} \frac{\langle N\rangle^{s} M}{\left\langle M^{2}+L\right\rangle^{\frac{1}{2}}} \sup _{\|w\|_{L^{2}}=1} I\right) \\
& \lesssim\left(\sum_{N_{1} \geq 1} \sum_{M_{1} \lesssim N_{1}} \sum_{N_{2} \lesssim N_{1}} \sum_{M_{2} \lesssim N_{2}} N_{1}^{-2 \epsilon}\left(M_{1} M_{2}\right)^{\frac{\epsilon}{2}}\right)^{\frac{1}{2}} \\
& \quad \times\left\{\sum_{N_{1}} \sum_{M_{1}}\left(\sum_{L_{1}}\left\|f_{N_{1}, M_{1}, L_{1}}\right\|_{L_{t x y}^{2}}\right)^{2}\right\}^{\frac{1}{2}}\left\{\sum_{N_{2}} \sum_{M_{2}}\left(\sum_{L_{2}}\left\|g_{N_{2}, M_{2}, L_{2}}\right\|_{L_{t x y}^{2}}\right)^{2}\right\}^{\frac{1}{2}} \\
& \lesssim\|u\|_{X^{s, \frac{1-\delta}{2}, 1}\|v\|_{X^{s, \frac{1-\delta}{2}, 1}} .}
\end{aligned}
$$


Now, we prove (3.18).

Case 1: $N_{1} \sim N_{2} \gg N, N_{1} \geq 1$.

We note that $M \lesssim \max \left\{M_{1}, M_{2}\right\}$ since $\xi+\eta=\left(\xi_{1}+\eta_{1}\right)+\left(\xi-\xi_{1}+\eta-\eta_{1}\right)$. By the symmetry, we can assume $M \lesssim M_{1}$. By the Hölder inequality, we have

$$
I \lesssim\left\|u_{N_{1}, M_{1}, L_{1}}\right\|_{L_{t x y}^{\frac{2}{1-\delta}}}\left\|v_{N_{2}, M_{2}, L_{2}} \cdot w_{N, M, L}\right\|_{L_{t x y}^{1+\delta}} \cdot
$$

Furthermore, we have

$$
\left\|u_{N_{1}, M_{1}, L_{1}}\right\|_{L_{t x y}^{\frac{2}{1-\delta}}} \lesssim L_{1}^{\frac{5}{6}}\left\|u_{N_{1}, M_{1}, L_{1}}\right\|_{L_{t x y}^{2}} \lesssim \frac{N_{1}^{-s}}{\left\langle M_{1}^{2}+L_{1}\right\rangle^{\frac{1-\delta}{2}-\frac{5}{6} \delta}}\left\|f_{N_{1}, M_{1}, L_{1}}\right\|_{L_{t x y}^{2}}
$$

by (3.16), and we have

$$
\begin{aligned}
& \left\|v_{N_{2}, M_{2}, L_{2}} \cdot w_{N, M, L}\right\|_{L_{t x y}^{\frac{2}{1+\delta}}} \\
& \lesssim J_{\delta, \epsilon}\left(N_{2}, M_{2}, N, M\right)\left(L_{2} L\right)^{\frac{1-\delta}{2}-\frac{\epsilon}{4}}\left\|v_{N_{2}, M_{2}, L_{2}}\right\|_{L_{t x y}^{2}}\left\|w_{N, M, L}\right\|_{L_{t x y}^{2}} \\
& \lesssim\left(M_{1} M_{2}\right)^{\frac{\epsilon}{4}} N_{2}^{-s-1+\delta+\frac{5}{4} \epsilon} N^{\frac{1-\delta}{2}-\frac{\epsilon}{4}} L^{\frac{1-\delta}{2}-\frac{\epsilon}{4}}\left\|g_{N_{2}, M_{2}, L_{2}}\right\|_{L_{t x y}^{2}}\left\|w_{N, M, L}\right\|_{L_{t x y}^{2}}
\end{aligned}
$$

by (3.13) and $M \lesssim M_{1}$. Therefore, if we choose $\epsilon>0$ as $\epsilon=\frac{10}{3} \delta$, we obtain

$$
\begin{aligned}
& \sum_{N \ll N_{1}} \sum_{M \lesssim M_{1}} \sum_{L} \frac{\langle N\rangle^{s} M}{\left\langle M^{2}+L\right\rangle^{\frac{1}{2}}} \sup _{\|w\|_{L^{2}=1}} I \\
& \lesssim\left(M_{1} M_{2}\right)^{\frac{\epsilon}{4}} N_{1}^{-s} N_{2}^{-s-1+\delta+\frac{5}{4} \epsilon}\left\|f_{N_{1}, M_{1}, L_{1}}\right\|_{L_{t x y}^{2}}\left\|g_{N_{2}, M_{2}, L_{2}}\right\|_{L_{t x y}^{2}} \\
& \quad \times\left(\sum_{N \ll N_{1}}\langle N\rangle^{s} N^{\frac{1-\delta}{2}-\frac{\epsilon}{4}} \sum_{M \lesssim M_{1}} \frac{M}{\left\langle M_{1}^{2}+L_{1}\right\rangle^{\frac{1-\delta}{2}-\frac{5}{6} \delta}} \sum_{L} \frac{L^{\frac{1-\delta}{2}-\frac{\epsilon}{4}}}{\left\langle M^{2}+L\right\rangle^{\frac{1}{2}}}\right) \\
& \lesssim N_{1}^{-\epsilon}\left(M_{1} M_{2}\right)^{\frac{\epsilon}{4}} N_{1}^{-s-\frac{1}{2}+\frac{\delta}{2}+2 \epsilon}\left\|f_{N_{1}, M_{1}, L_{1}}\right\|_{L_{t x y}^{2}}\left\|g_{N_{2}, M_{2}, L_{2}}\right\|_{L_{t x y}^{2}}
\end{aligned}
$$

for $s \geq-\frac{1-\delta}{2}+\frac{\epsilon}{4}$ since

$$
\sum_{M \lesssim M_{1}} \frac{M}{\left\langle M_{1}^{2}+L_{1}\right\rangle^{\frac{1-\delta}{2}-\frac{5}{6} \delta}} \sum_{L} \frac{L^{\frac{1-\delta}{2}-\frac{\epsilon}{4}}}{\left\langle M^{2}+L\right\rangle^{\frac{1}{2}}} \lesssim \sum_{M \lesssim M_{1}} \frac{M^{1-\delta-\frac{\epsilon}{2}}}{\left\langle M_{1}\right\rangle^{1-\frac{8}{3} \delta}} \lesssim 1 .
$$

As a result, we get (3.18) for $s>-\frac{1}{2}$ if we choose $\delta>0$ as $0<\delta<\frac{6}{43}\left(s+\frac{1}{2}\right)$.

Case 2: $N \sim N_{1} \gg N_{2}, N_{1} \geq 1$.

By the Hölder inequality, we have

$$
I \lesssim\left\|u_{N_{1}, M_{1}, L_{1}} \cdot v_{N_{2}, M_{2}, L_{2}}\right\|_{L_{t x y}^{\frac{2}{1+\delta}}}\left\|w_{N, M, L}\right\|_{L_{t x y}^{\frac{2}{1-\delta}}} \cdot
$$

Furthermore, we have

$$
\left\|w_{N, M, L}\right\|_{L_{t x y}^{\frac{2}{1-\delta}}} \lesssim L^{\frac{5}{6} \delta}\left\|w_{N, M, L}\right\|_{L_{t x y}^{2}}
$$


by (3.16), and we have

$$
\begin{aligned}
& \left\|u_{N_{1}, M_{1}, L_{1}} \cdot v_{N_{2}, M_{2}, L_{2}}\right\|_{L_{t x y}^{\frac{2}{1+\delta}}} \\
& \lesssim J_{\delta, \epsilon}\left(N_{1}, M_{1}, N_{2}, M_{2}\right)\left(L_{1} L_{2}\right)^{\frac{1-\delta}{2}-\frac{\epsilon}{4}}\left\|u_{N_{1}, M_{1}, L_{1}}\right\|_{L_{t x y}^{2}}\left\|v_{N_{2}, M_{2}, L_{2}}\right\|_{L_{t x y}^{2}} \\
& \lesssim\left(M_{1} M_{2}\right)^{\frac{\epsilon}{4}} N_{1}^{-s-1+\delta+\frac{5}{4} \epsilon}\left\langle N_{2}\right\rangle^{-s} N_{2}^{\frac{1-\delta}{2}-\frac{\epsilon}{4}}\left\|f_{N_{1}, M_{1}, L_{1}}\right\|_{L_{t x y}^{2}}\left\|g_{N_{2}, M_{2}, L_{2}}\right\|_{L_{t x y}^{2}}
\end{aligned}
$$

by (3.13). Therefore, if $s \leq \frac{1-\delta}{2}-\frac{\epsilon}{4}$, we obtain

$$
\begin{aligned}
& \sum_{N \sim N_{1}} \sum_{M \lesssim N} \sum_{L} \frac{\langle N\rangle^{s} M}{\left\langle M^{2}+L\right\rangle^{\frac{1}{2}}} \sup _{\|w\|_{L^{2}}=1} I \\
& \lesssim\left(M_{1} M_{2}\right)^{\frac{\epsilon}{4}} N_{1}^{-s-\frac{1-\delta}{2}+\epsilon}\left\|f_{N_{1}, M_{1}, L_{1}}\right\|_{L_{t x y}^{2}}\left\|g_{N_{2}, M_{2}, L_{2}}\right\|_{L_{t x y}^{2}}\left(\sum_{M \lesssim N_{1}} M \sum_{L} \frac{L^{\frac{5}{6}} \delta}{\left\langle M^{2}+L\right\rangle^{\frac{1}{2}}}\right) \\
& \lesssim N_{1}^{-\epsilon}\left(M_{1} M_{2}\right)^{\frac{\epsilon}{4}} N_{1}^{-s-\frac{1}{2}+\frac{13}{6} \delta+2 \epsilon}\left\|f_{N_{1}, M_{1}, L_{1}}\right\|_{L_{t x y}^{2}}\left\|g_{N_{2}, M_{2}, L_{2}}\right\|_{L_{t x y}^{2}},
\end{aligned}
$$

since

$$
\sum_{L} \frac{L^{\frac{5}{6} \delta}}{\left\langle M^{2}+L\right\rangle^{\frac{1}{2}}} \lesssim M^{-\left(1-\frac{5}{3} \delta\right)} .
$$

As a result, we get (3.18) for $\frac{1}{2}>s>-\frac{1}{2}$ if we choose $\delta>0$ and $\epsilon>0$ as $0<\epsilon<\frac{1}{2}\left(s+\frac{1}{2}\right), 0<\delta<\min \left\{\frac{6}{13}\left(s+\frac{1}{2}-2 \epsilon\right), 2\left(\frac{1}{2}-s-\frac{\epsilon}{2}\right)\right\}$.

While if $s \geq \frac{1}{2}$, then we have

$$
I \lesssim\left(M_{1} M_{2}\right)^{\frac{\epsilon}{4}} N_{1}^{-s-\frac{1-\delta}{2}+\epsilon} L^{\frac{5}{6} \delta}\left\|f_{N_{1}, M_{1}, L_{1}}\right\|_{L_{t x y}^{2}}\left\|g_{N_{2}, M_{2}, L_{2}}\right\|_{L_{t x y}^{2}}\left\|w_{N, M, L}\right\|_{L_{t x y}^{2}}
$$

by the same argument with using $\left\langle N_{2}\right\rangle^{-s} \lesssim 1$. Therefore, we obtain

$$
\begin{aligned}
& \sum_{N \sim N_{1}} \sum_{M \lesssim N} \sum_{L} \frac{\langle N\rangle^{s} M}{\left\langle M^{2}+L\right\rangle^{\frac{1}{2}}} \sup _{\|w\|_{L^{2}}=1} I \\
& \lesssim N_{1}^{-\epsilon}\left(M_{1} M_{2}\right)^{\frac{\epsilon}{4}} N_{1}^{-\frac{1}{2}+\frac{13}{6} \delta+2 \epsilon}\left\|f_{N_{1}, M_{1}, L_{1}}\right\|_{L_{t x y}^{2}}\left\|g_{N_{2}, M_{2}, L_{2}}\right\|_{L_{t x y}^{2}},
\end{aligned}
$$

which implies (3.18) since $-\frac{1}{2}+\frac{13}{6} \delta+2 \epsilon<0$.

Case 3: $N \sim N_{1} \sim N_{2} \geq 1$ into

We can assume $M \lesssim M_{1}$ such as Case 1 . We split $v_{N_{2}, M_{2}, L_{2}}$ and $w_{N, M, L}$

$$
v_{N_{2}, M_{2}, L_{2}}=\sum_{i=1}^{3} R_{i} v_{N_{2}, M_{2}, L_{2}}, \quad w_{N, M, L}=\sum_{j=1}^{3} R_{j} w_{N, M, L} .
$$

We put

$$
I_{i, j}=\left|\int u_{N_{1}, M_{1}, L_{1}} \cdot R_{i} v_{N_{2}, M_{2}, L_{2}} \cdot R_{j} w_{N, M, L} d t d x d y\right|,
$$

where $R_{i}(i=1,2,3)$ are projections given by

$$
\mathcal{F}_{x y}\left[R_{1} f\right]=\mathbf{1}_{\{|\xi| \gg|\eta|\}} \widehat{f}, \mathcal{F}_{x y}\left[R_{2} f\right]=\mathbf{1}_{\{|\xi| \sim|\eta|\}} \widehat{f}, \mathcal{F}_{x y}\left[R_{3} f\right]=\mathbf{1}_{\{|\xi| \ll|\eta|\}} \widehat{f} .
$$


We note that $\mathcal{F}_{x y}\left[w_{N, M, L}\right]$ is supported in at least one of $\{(\xi, \eta)|| \xi \mid \sim N\}$ or $\{(\xi, \eta)|| \eta \mid \sim N\}$. By the symmetry, we can assume $\operatorname{supp} \mathcal{F}_{x y}\left[w_{N, M, L}\right] \subset$ $\{(\xi, \eta)|| \xi \mid \sim N\}$. Then, it suffice to show the estimate for $I_{i, j}$ with $i=1,2,3$, $j=1,2$.

Estimate for $I_{1,1}$

In this case, we note that $N \sim N_{1} \sim N_{2} \sim M \sim M_{1} \sim M_{2}$ and

$$
\left|\xi \xi_{1} \xi_{2}+\eta \eta_{1} \eta_{2}\right| \sim\left|\xi \xi_{1} \xi_{2}\right| \sim N_{1}^{3}
$$

for $\left(\xi_{1}, \eta_{1}\right) \in \operatorname{supp} \mathcal{F}_{x y}\left[u_{N_{1}, M_{1}, L_{1}}\right],\left(\xi_{2}, \eta_{2}\right) \in \operatorname{supp} \mathcal{F}_{x y}\left[v_{N_{2}, M_{2}, L_{2}}\right]$ with $\xi_{1}+\xi_{2}=$ $\xi, \eta_{1}+\eta_{2}=\eta$. It implies

$$
\max \left\{L_{1}, L_{2}, L\right\} \gtrsim N_{1}^{3}
$$

since

$$
\left|\left(\tau_{1}-\xi_{1}^{3}-\eta_{1}^{3}\right)+\left(\tau_{2}-\xi_{2}^{3}-\eta_{2}^{3}\right)-\left(\tau-\xi^{3}-\eta^{3}\right)\right|=3\left|\xi \xi_{1} \xi_{2}+\eta \eta_{1} \eta_{2}\right| .
$$

holds for $\left(\tau_{i}, \xi_{i}, \eta_{i}\right)(i=1,2)$ with $(\tau, \xi, \eta)=\left(\tau_{1}+\tau_{2}, \xi_{1}+\xi_{2}, \eta_{1}+\eta_{2}\right)$.

(i) For the case $L \gtrsim N_{1}^{3}$

By the Hölder inequality, (3.3), and (3.16), we have

$$
\begin{aligned}
I & \lesssim\left\|u_{N_{1}, M_{1}, L_{1}}\right\|_{L_{t x y}^{\frac{4}{1+\delta}}}\left\|v_{N_{2}, M_{2}, L_{2}}\right\|_{L_{t x y}^{\frac{4}{1+\delta}}}\left\|w_{N, M, L}\right\|_{L_{t x y}^{\frac{2}{1+\delta}}} \\
& \lesssim\left(N_{1} M_{1} N_{2} M_{2}\right)^{\frac{\epsilon}{4}}\left(L_{1} L_{2}\right)^{\frac{5(1-\delta)}{12}}-\frac{\epsilon}{6} L^{\frac{5}{6} \delta}\left\|u_{N_{1}, M_{1}, L_{1}}\right\|_{L_{t x y}^{2}}\left\|v_{N_{2}, M_{2}, L_{2}}\right\|_{L_{t x y}^{2}}\left\|w_{N, M, L}\right\|_{L_{t x y}^{2}} \\
& \sim N_{1}^{-\epsilon}\left(M_{1} M_{2}\right)^{\frac{\epsilon}{4}} N_{1}^{-2 s+\frac{3}{2} \epsilon} L^{\frac{5}{6} \delta}\left\|f_{N_{1}, M_{1}, L_{1}}\right\|_{L_{t x y}^{2}}\left\|g_{N_{2}, M_{2}, L_{2}}\right\|_{L_{t x y}^{2}}\left\|w_{N, M, L}\right\|_{L_{t x y}^{2} .}
\end{aligned}
$$

Therefore, we obtain

$$
\begin{aligned}
& \sum_{N \sim N_{1}} \sum_{M \lesssim N} \sum_{L \gtrsim N_{1}^{3}} \frac{\langle N\rangle^{s} M}{\left\langle M^{2}+L\right\rangle^{\frac{1}{2}}} \sup _{\|w\|_{L^{2}=1}} I \\
& \lesssim N_{1}^{-\epsilon}\left(M_{1} M_{2}\right)^{\frac{\epsilon}{4}} N_{1}^{-s+\frac{3}{2} \epsilon}\left\|f_{N_{1}, M_{1}, L_{1}}\right\|_{L_{t x y}^{2}}\left\|g_{N_{2}, M_{2}, L_{2}}\right\|_{L_{t x y}^{2}}\left(\sum_{M \lesssim N_{1}} M \sum_{L \gtrsim N_{1}^{3}} \frac{L^{\frac{5}{6}} \delta}{\left\langle M^{2}+L\right\rangle^{\frac{1}{2}}}\right) \\
& \lesssim N_{1}^{-\epsilon}\left(M_{1} M_{2}\right)^{\frac{\epsilon}{4}} N_{1}^{-s-\frac{1}{2}+\frac{5}{2} \delta+\frac{3}{2} \epsilon}\left\|f_{N_{1}, M_{1}, L_{1}}\right\|_{L_{t x y}^{2}}\left\|g_{N_{2}, M_{2}, L_{2}}\right\|_{L_{t x y}^{2}},
\end{aligned}
$$

since

$$
\sum_{L \gtrsim N_{1}^{3}} \frac{L^{\frac{5}{6} \delta}}{\left\langle M^{2}+L\right\rangle^{\frac{1}{2}}} \lesssim \sum_{L \gtrsim N_{1}^{3}} L^{-\left(\frac{1}{2}-\frac{5}{6} \delta\right)} \lesssim N_{1}^{-\frac{3}{2}+\frac{5}{2} \delta} .
$$

As a result, we get (3.18) for $s>-\frac{1}{2}$ if we choose $\delta>0$ and $\epsilon>0$ as $0<\epsilon<\frac{2}{3}\left(s+\frac{1}{2}\right), 0<\delta<\frac{2}{5}\left(s+\frac{1}{2}-\frac{3}{2} \epsilon\right)$.

(ii) For the case $L_{1} \gtrsim N_{1}^{3}$

By the Hölder inequality, (3.16), and (3.3), we have

$$
\begin{aligned}
I & \lesssim\left\|u_{N_{1}, M_{1}, L_{1}}\right\|_{L_{t x y}^{\frac{2}{1-\delta}}}\left\|v_{N_{2}, M_{2}, L_{2}}\right\|_{L_{t x y}^{\frac{4}{1+\delta}}}\left\|w_{N, M, L}\right\|_{L_{t x y}^{\frac{4}{1+\delta}}} \\
& \lesssim L_{1}^{\frac{5}{6} \delta}\left(N_{2} M_{2} N M\right)^{\frac{\epsilon}{4}}\left(L_{2} L\right)^{\frac{5(1-\delta)}{12}}-\frac{\epsilon}{6}\left\|u_{N_{1}, M_{1}, L_{1}}\right\|_{L_{t x y}^{2}}\left\|v_{N_{2}, M_{2}, L_{2}}\right\|_{L_{t x y}^{2}}\left\|w_{N, M, L}\right\|_{L_{t x y}^{2}} \\
& \lesssim N_{1}^{-\epsilon}\left(M_{1} M_{2}\right)^{\frac{\epsilon}{4}} N_{1}^{-2 s-\frac{3}{2}+4 \delta+\frac{3}{2} \epsilon} L^{\frac{5(1-\delta)}{12}-\frac{\epsilon}{6}}\left\|f_{N_{1}, M_{1}, L_{1}}\right\|_{L_{t x y}^{2}}\left\|g_{N_{2}, M_{2}, L_{2}}\right\|_{L_{t x y}^{2}}\left\|w_{N, M, L}\right\|_{L_{t x y}^{2}}
\end{aligned}
$$


since $L_{1}^{\frac{5}{6} \delta}\left\langle M_{1}^{2}+L_{1}\right\rangle^{-\frac{1-\delta}{2}} \lesssim L_{1}^{-\left(\frac{1}{2}-\frac{4}{3} \delta\right)} \lesssim N_{1}^{-\frac{3}{2}+4 \delta}$. Therefore, we obtain

$$
\begin{aligned}
& \sum_{N \sim N_{1}} \sum_{M \lesssim N} \sum_{L} \frac{\langle N\rangle^{s} M}{\left\langle M^{2}+L\right\rangle^{\frac{1}{2}}} \sup _{\|w\|_{L^{2}}=1} I \\
& \lesssim N_{1}^{-\epsilon}\left(M_{1} M_{2}\right)^{\frac{\epsilon}{4}} N_{1}^{-s-\frac{3}{2}+4 \delta+\frac{3}{2} \epsilon}\left\|f_{N_{1}, M_{1}, L_{1}}\right\|_{L_{t x y}^{2}}\left\|g_{N_{2}, M_{2}, L_{2}}\right\|_{L_{t x y}^{2}}\left(\sum_{M \lesssim N_{1}} M \sum_{L} \frac{L^{\frac{5(1-\delta)-2 \epsilon}{12}}}{\left\langle M^{2}+L\right\rangle^{\frac{1}{2}}}\right) \\
& \lesssim N_{1}^{-\epsilon}\left(M_{1} M_{2}\right)^{\frac{\epsilon}{4}} N_{1}^{-s-\frac{2}{3}+\frac{19}{6} \delta+\frac{7}{6} \epsilon}\left\|f_{N_{1}, M_{1}, L_{1}}\right\|_{L_{t x y}^{2}}\left\|g_{N_{2}, M_{2}, L_{2}}\right\|_{L_{t x y}^{2}},
\end{aligned}
$$

since

$$
\sum_{L} \frac{L^{\frac{5(1-\delta)-2 \epsilon}{12}}}{\left\langle M^{2}+L\right\rangle^{\frac{1}{2}}} \lesssim M^{-\frac{1+5 \delta+2 \epsilon}{6}} .
$$

As a result, we get (3.18) for $s>-\frac{2}{3}$ if we choose $\delta>0$ and $\epsilon>0$ as $0<\epsilon<\frac{6}{7}\left(s+\frac{1}{2}\right), 0<\delta<\frac{6}{19}\left(s+\frac{2}{3}-\frac{7}{6} \epsilon\right)$. The case $L_{2} \gtrsim N^{3}$ is same.

Estimate for $I_{2,2}$

In this case, we have

$$
\left|\xi_{2}\right| \sim\left|\eta_{2}\right| \sim N_{2},|\xi| \sim|\eta| \sim N
$$

By the Hölder inequality, (3.16), (3.4), and $M \lesssim M_{1}$, we have

$$
\begin{aligned}
I & \lesssim\left\|u_{N_{1}, M_{1}, L_{1}}\right\|_{L_{t x y}^{\frac{2}{1-\delta}}}\left\|v_{N_{2}, M_{2}, L_{2}}\right\|_{L_{t x y}^{\frac{4}{1+\delta}}}\left\|w_{N, M, L}\right\|_{L_{t x y}^{\frac{4}{1+\delta}}} \\
& \lesssim L_{1}^{\frac{5}{6} \delta}\left(N_{2} M_{2} N M\right)^{\frac{\epsilon}{4}}\left(N_{2} N\right)^{-\frac{1-\delta-\epsilon}{4}}\left(L_{2} L\right)^{\frac{1-\delta}{2}-\frac{\epsilon}{4}}\left\|u_{N_{1}, M_{1}, L_{1}}\right\|_{L_{t x y}^{2}}\left\|v_{N_{2}, M_{2}, L_{2}}\right\|_{L_{t x y}^{2}}\left\|w_{N, M, L}\right\|_{L_{t x y}^{2}} \\
& \lesssim\left(M_{1} M_{2}\right)^{\frac{\epsilon}{4}} N_{1}^{-2 s-\frac{1-\delta}{2}+\epsilon} \frac{L^{\frac{1-\delta}{2}-\frac{\epsilon}{4}}}{\left\langle M_{1}^{2}+L_{1}\right\rangle^{\frac{1-\delta}{2}-\frac{5}{6} \delta}}\left\|f_{N_{1}, M_{1}, L_{1}}\right\|_{L_{t x y}^{2}}\left\|g_{N_{2}, M_{2}, L_{2}}\right\|_{L_{t x y}^{2}}\left\|w_{N, M, L}\right\|_{L_{t x y}^{2}} .
\end{aligned}
$$

Therefore, we get (3.18) for $s>-\frac{1}{2}$ by the same argument as in Case 1 .

Estimate for $I_{1,2}$

In this case, we have

$$
\left|\eta_{2}^{2}-\eta^{2}\right| \sim|\eta|^{2} \sim N^{2}
$$

Therefore, we obtain

$\left\|R_{1} v_{N_{2}, M_{2}, L_{2}} \cdot R_{2} w_{N, M, L}\right\|_{L_{t x y}^{2}} \lesssim N^{-\frac{1}{2}} L_{2}^{\frac{1}{2}} L^{\frac{1}{2}}\left\|R_{1} v_{N_{2}, M_{2}, L_{2}}\right\|_{L_{t x y}^{2}}\left\|R_{2} w_{N, M, L}\right\|_{L_{t x y}^{2}}$

by (3.8) since

$$
R_{1} v_{N_{2}, M_{2}, L_{2}} \cdot R_{2} w_{N, M, L}=R_{K}^{(2)}\left(R_{1} v_{N_{2}, M_{2}, L_{2}} \cdot R_{2} w_{N, M, L}\right)
$$

with $K \sim N$ holds. While, by the Cauchy-Schwartz inequality, we have

$$
\left\|R_{1} u_{N_{1}, M_{1}, L_{1}} \cdot R_{j} v_{N_{2}, M_{2}, L_{2}}\right\|_{L_{t x y}^{1}} \lesssim\left\|R_{1} v_{N_{2}, M_{2}, L_{2}}\right\|_{L_{t x y}^{2}}\left\|R_{2} w_{N, M, L}\right\|_{L_{t x y}^{2}} .
$$

Therefore, we obtain the bilinear Stirchartz estimate such as (3.13) for the product $R_{1} v_{N_{2}, M_{2}, L_{2}} \cdot R_{2} w_{N, M, L}$, and we get (3.18) for $s>-\frac{1}{2}$ by the same argument as in Case 1 since $M \lesssim M_{1}$. The estimates for $I_{2,1}, I_{3,1}$, and $I_{3,2}$ are obtained by the same way. 
Remark 3.9. We can also obtain the bilinear estimate

$\left\|\left(\partial_{x}+\partial_{y}\right)(u v)\right\|_{X^{s,-\frac{1}{2}, 1}} \leq \frac{C_{3}}{2}\left(\|u\|_{X^{s, \frac{1-\delta}{2}, 1}}\|v\|_{X^{s_{0}, \frac{1-\delta}{2}, 1}}+\|u\|_{X^{s_{0}, \frac{1-\delta}{2}, 1}}\|v\|_{X^{s, \frac{1-\delta}{2}, 1}}\right)$

for $s \geq s_{0}>-\frac{1}{2}$ by using

$$
\langle\xi\rangle^{s} \lesssim\langle\xi\rangle^{s_{0}}\left(\left\langle\xi_{1}\right\rangle^{s-s_{0}}+\left\langle\xi-\xi_{1}\right\rangle^{s-s_{0}}\right) .
$$

\section{Proof of the well-posedness}

In this section, we prove Theorem 1.1 and 1.2. For $T>0$ and $v_{0} \in H^{s}\left(\mathbb{R}^{2}\right)$, we define the map $\Phi_{T, v_{0}}$ as

$$
\Phi_{T, v_{0}}(v)(t):=\psi(t)\left(W(t) u_{0}+\int_{0}^{t} W\left(t-t^{\prime}\right)\left(\partial_{x}+\partial_{y}\right)\left(\psi_{T}\left(t^{\prime}\right)^{2} v\left(t^{\prime}\right)^{2}\right) d t^{\prime}\right),
$$

where $\psi$ is cut-off function defined in Section 2, and $\psi_{T}(t)=\psi\left(\frac{t}{T}\right)$. For $R>0$ and Banach space $X$, we define $B_{R}(X):=\left\{u \in X \mid\|u\|_{X} \leq R\right\}$. To obtain the well-posedness of (1.6) in $H^{s}\left(\mathbb{R}^{2}\right)$, we prove that $\Phi_{T, v_{0}}$ is a contraction map on closed subset of $X^{s, \frac{1}{2}, 1}$.

Lemma 4.1. Let $0<T \leq 1,0<\delta \leq 1$. There exist $C_{4}>0$ and $\mu=\mu(\delta)>0$, such that for any $u \in X^{s, \frac{1}{2}, 1}$, we have

$$
\left\|\psi_{T} u\right\|_{X^{s, \frac{1-\delta}{2}, 1}} \leq C_{4} T^{\mu}\|u\|_{X^{s, \frac{1}{2}, 1}} .
$$

The proof of Lemma 4.1 is almost same as the proof of Lemma 2.5 and 3.1 in 8 .

Proof of Theorem 1.1. Let $s \geq s_{0}>-\frac{1}{2}$ and $v_{0} \in H^{s}\left(\mathbb{R}^{2}\right)$ are given, and $T \in$ $(0,1], R>0$ will be chosen later. We define the function space $Z^{s}$ as

$$
Z^{s}:=\left\{v \in X^{s, \frac{1}{2}, 1} \mid\|v\|_{Z^{s}}:=\|v\|_{X^{s_{0}, \frac{1}{2}, 1}}+\alpha\|v\|_{X^{s, \frac{1}{2}, 1}}<\infty\right\},
$$

where $\alpha=\left\|v_{0}\right\|_{H^{s_{0}}} /\left\|v_{0}\right\|_{H^{s}}$. For $v, v_{1}, v_{2} \in B_{R}\left(Z^{s}\right)$, we have

$$
\begin{aligned}
\left\|\Phi_{T, v_{0}}(v)\right\|_{Z^{s}} & \leq C_{1}(1+\alpha)\left\|v_{0}\right\|_{H^{s_{0}}}+C_{2} C_{3} C_{4}^{2} T^{2 \mu}\|v\|_{Z^{s}}^{2} \\
& \leq C_{1}(1+\alpha)\left\|v_{0}\right\|_{H^{s_{0}}}+C_{2} C_{3} C_{4}^{2} T^{2 \mu} R^{2}
\end{aligned}
$$

and

$$
\begin{aligned}
\left\|\Phi_{T, v_{0}}\left(v_{1}\right)-\Phi_{T, v_{0}}\left(v_{2}\right)\right\|_{Z^{s}} & \leq C_{2} C_{3} C_{4}^{2} T^{2 \mu}\left\|v_{1}+v_{2}\right\|_{Z^{s}}\left\|v_{1}-v_{2}\right\|_{Z^{s}} \\
& \leq C_{2} C_{3} C_{4}^{2} T^{2 \mu} R\left\|v_{1}-v_{2}\right\|_{Z^{s}}
\end{aligned}
$$

by Proposition 2.3, 2.4, 3.1, Remark 3.9, and Lemma 4.1. Therefore, if we choose $T, R$ as

$$
R=2 C_{1}(1+\alpha)\left\|v_{0}\right\|_{H^{s_{0}}}, 0<T^{2 \mu}<\left(4 C_{1} C_{2} C_{3} C_{4}^{2}(1+\alpha)\left\|v_{0}\right\|_{H^{s_{0}}}\right)^{-1},
$$

then $\Phi_{T, v_{0}}$ is contraction map on $B_{R}\left(Z^{s}\right)$. We note that $T=T\left(\left\|v_{0}\right\|_{H^{s_{0}}}\right)$. By Banach's fixed point theorem, there exists a solution $v \in X^{s, \frac{1}{2}, 1}$ to $v(t)=$ $\Phi_{T, v_{0}}(v)(t)$ and $\left.v\right|_{[0, T]} \in X_{T}^{s, \frac{1}{2}, 1}$ satisfies (2.1) on $[0, T]$. The Lipschitz continuous dependence on initial data is obtained by the similar argument as above. The uniqueness is obtained by the same argument as in Section 4.2 of [20]. 
Next, to prove the global well-posedness of (1.6) in $\widetilde{H}^{s}\left(\mathbb{R}^{2}\right)$, we define the function space $\widetilde{X}^{s, b, 1}$ as the completion of the Schwartz class $\mathcal{S}\left(\mathbb{R}_{t} \times \mathbb{R}_{x, y}^{2}\right)$ with the norm

$$
\|u\|_{\widetilde{X}^{s, b, 1}}=\left\{\sum_{N \in 2^{\mathbb{Z}}} \sum_{M \in 2^{\mathbb{Z}}}\left(\sum_{L \in 2^{\mathbb{Z}}}\langle M\rangle^{s}\left\langle M^{2}+L\right\rangle^{b}\left\|P_{N, M} Q_{L} u\right\|_{L_{t x y}^{2}}\right)^{2}\right\}^{\frac{1}{2}} .
$$

We also define $\widetilde{X}_{T}^{s, b, 1}$ as the time localized space of $\widetilde{X}^{s, b, 1}$.

Remark 4.2. We can see that $\widetilde{X}_{T}^{s \frac{1}{2}, 1} \hookrightarrow L^{2}\left((0, T) ; \widetilde{H}^{s+1}\left(\mathbb{R}^{2}\right)\right)$ since $\langle M\rangle^{s+1} \lesssim$ $\langle M\rangle^{s}\left\langle M^{2}+L\right\rangle^{\frac{1}{2}}$ and $l_{L}^{1} \hookrightarrow l_{L}^{2}$ hold.

Proposition 4.3. Let $s \in \mathbb{R}$. There exists $C_{1}>0$, such that for any $u_{0} \in$ $\widetilde{H}^{s}\left(\mathbb{R}^{2}\right)$, we have

$$
\left\|\psi(t) W(t) u_{0}\right\|_{\widetilde{X}^{s, \frac{1}{2}, 1}} \leq C_{1}\left\|u_{0}\right\|_{\widetilde{H}^{s}} .
$$

Proposition 4.4. Let $s \in \mathbb{R}$. There exists $C_{2}>0$, such that for any $F \in$ $\widetilde{X}^{s,-\frac{1}{2}, 1}$, we have

$$
\|\psi(t) \mathcal{L} F(t)\|_{\tilde{X}^{s, \frac{1}{2}, 1}} \leq C_{2}\|F\|_{\tilde{X}^{s,-\frac{1}{2}, 1}}
$$

The proof of Proposition 4.3 and 4.4 are same as the proof of Proposition 2.3 and 2.4.

Proposition 4.5. Let $s>-\frac{1}{2}$. There exist $0<\delta \ll 1$ and $C_{3}>0$, such that for any $u, v \in \widetilde{X}^{s, \frac{1-\delta}{2}, 1}$, we have

$$
\left\|\left(\partial_{x}+\partial_{y}\right)(u v)\right\|_{\tilde{X}^{s,-\frac{1}{2}, 1}} \leq C_{3}\|u\|_{\widetilde{X}^{s, \frac{1-\delta}{2}, 1}}\|v\|_{\widetilde{X}^{s, \frac{1-\delta}{2}, 1}} .
$$

The proof of Proposition 4.5 is similar to the proof of Proposition 3.1. We will give the proof at the last part of this section.

Proof of Theorem [1.2. Let $s \geq s_{0}>-\frac{1}{2}$ are given. By Proposition 4.3 4.4, 4.5, and using the same argument as in the proof of Theorem 1.1. we obtain the solution $v \in \widetilde{X}_{T}^{s, \frac{1}{2}, 1}$ to (1.6) on $[0, T]$ with $T=T\left(\left\|v_{0}\right\|_{\widetilde{H}^{s_{0}}}\right)$. Let $T^{\prime} \in(0, T)$ be fixed. Since $\widetilde{X}_{T}^{s, \frac{1}{2}, 1} \hookrightarrow L^{2}\left([0, T] ; \widetilde{H}^{s+1}\left(\mathbb{R}^{2}\right)\right)$ holds, there exists $t_{0} \in\left(0, T^{\prime}\right)$ such that $v\left(t_{0}\right) \in \widetilde{H}^{s+1}\left(\mathbb{R}^{2}\right)$. Therefore, by choosing $v\left(t_{0}\right)$ as the initial data and using the uniqueness of the solution, we obtain $v\left(t_{0}+\cdot\right) \in \widetilde{X}_{T-t_{0}}^{s+1, \frac{1}{2}, 1}$. In particular, we have $v\left(T^{\prime}\right) \in \widetilde{H}^{s+1}\left(\mathbb{R}^{2}\right)$. By repeating this argument, we get $v\left(T^{\prime}\right) \in \widetilde{H}^{\infty}\left(\mathbb{R}^{2}\right)$. Since we can choose $T^{\prime}>0$ arbitrary small, $v$ belongs to $C\left((0, T] ; \widetilde{H}^{\infty}\left(\mathbb{R}^{2}\right)\right)$. This arrows us to take the $L^{2}$-scalar product of (1.6) with $v$, and we have

$$
\begin{aligned}
\frac{d}{d t}\|v(t)\|_{L_{x}^{2}}^{2} & =\left(\partial_{t} v(t), v(t)\right)_{L_{x}^{2}} \\
& =\left(-\left(\partial_{x}^{3}+\partial_{y}^{3}\right) v(t)+\left(\partial_{x}+\partial_{y}\right)^{2} v(t)+\left(\partial_{x}+\partial_{y}\right)\left(v(t)^{2}\right), v(t)\right)_{L_{x}^{2}} \\
& =-\left\|\left(\partial_{x}+\partial_{y}\right) v(t)\right\|_{L_{x}^{2}}^{2} \leq 0
\end{aligned}
$$

for any $t \in(0, T)$. Therefore, $\|v(t)\|_{L_{x}^{2}}$ is non-increasing, and we can extend the solution $v$ globally in time. 
Remark 4.6. We note that the embedding $X_{T}^{s, \frac{1}{2}, 1} \hookrightarrow L^{2}\left([0, T] ; H^{s+1}\left(\mathbb{R}^{2}\right)\right)$ does not hold. Therefore, we cannot use the above argument for initial data $v_{0} \in$ $H^{s}\left(\mathbb{R}^{2}\right)$.

Finally, we give the proof of Proposition 4.5

Proof of Proposition 4.5. We put

$$
u_{N_{1}, M_{1}, L_{1}}=P_{N_{1}, M_{1}} Q_{L_{1}} u, v_{N_{2}, M_{2}, L_{2}}=P_{N_{2}, M_{2}} Q_{L_{2}} v, w_{N, M, L}=P_{N, M} Q_{L} w,
$$

$f_{N_{1}, M_{1}, L_{1}}=\left\langle M_{1}\right\rangle^{s}\left\langle M_{1}^{2}+L_{1}\right\rangle^{\frac{1-\delta}{2}} u_{N_{1}, M_{1}, L_{1}}, g_{N_{2}, M_{2}, L_{2}}=\left\langle M_{2}\right\rangle^{s}\left\langle M_{2}^{2}+L_{2}\right\rangle^{\frac{1-\delta}{2}} v_{N_{2}, M_{2}, L_{2}}$ for $0<\delta \ll 1$ and

$$
I=\left|\int u_{N_{1}, M_{1}, L_{1}} \cdot v_{N_{2}, M_{2}, L_{2}} \cdot w_{N, M, L} d t d x d y\right| .
$$

We use $L_{1}^{b}\left\|u_{N_{1}, M_{1}, L_{1}}\right\|_{L_{t x y}^{2}} \lesssim\left\langle M_{1}\right\rangle^{-s}\left\|f_{N_{1}, M_{1}, L_{1}}\right\|_{L_{t x y}^{2}}$ and $L_{2}^{b}\left\|v_{N_{2}, M_{2}, L_{2}}\right\|_{L_{t x y}^{2}} \lesssim$ $\left\langle M_{2}\right\rangle^{-s}\left\|g_{N_{2}, M_{2}, L_{2}}\right\|_{L_{t x y}^{2}}$ instead of $L_{1}^{b}\left\|u_{N_{1}, M_{1}, L_{1}}\right\|_{L_{t x y}^{2}} \lesssim\left\langle N_{1}\right\rangle^{-s}\left\|f_{N_{1}, M_{1}, L_{1}}\right\|_{L_{t x y}^{2}}$ and $L_{2}^{b}\left\|v_{N_{2}, M_{2}, L_{2}}\right\|_{L_{t x y}^{2}} \lesssim\left\langle N_{2}\right\rangle^{-s}\left\|g_{N_{2}, M_{2}, L_{2}}\right\|_{L_{t x y}^{2}}$ in the proof of Proposition 3.1 By the same argument as in the proof of Proposition 3.1, we have

$$
\sum_{N_{1}, M_{1} \lesssim 1} \sum_{L_{1}} \sum_{N_{2}, M_{2} \lesssim 1} \sum_{L_{2}}\left(\sum_{N, M, L} \frac{\langle M\rangle^{s} M}{\left\langle M^{2}+L\right\rangle^{\frac{1}{2}}} \sup _{\|w\|_{L^{2}}=1} I\right) \lesssim\|u\|_{\widetilde{X}^{s, \frac{1-\delta}{2}, 1}}\|v\|_{\widetilde{X}^{s, \frac{1-\delta}{2}, 1}}
$$

for any $s \in \mathbb{R}$ and it suffice to show that

$$
\begin{aligned}
& \sum_{N, M, L} \frac{\langle M\rangle^{s} M}{\left\langle M^{2}+L\right\rangle^{\frac{1}{2}}} \sup _{\|w\|_{L^{2}}=1} I \\
& \lesssim N_{1}^{-\epsilon}\left(M_{1} M_{2}\right)^{\frac{\epsilon}{4}}\left\|f_{N_{1}, M_{1}, L_{1}}\right\|_{L_{t x y}^{2}}\left\|g_{N_{2}, M_{2}, L_{2}}\right\|_{L_{t x y}^{2}}
\end{aligned}
$$

for $N_{1} \geq N_{2}, N_{1} \geq 1$, and small $\epsilon>0$.

Case 1': $N_{1} \sim N_{2} \gg N$

We only have to modify little in the proof of Proposition 3.1. Case 1. Since it hold that

$$
\left\langle M_{1}\right\rangle^{-s} \sum_{M \lesssim M_{1}} \frac{\langle M\rangle^{s} M}{\left\langle M_{1}^{2}+L_{1}\right\rangle^{\frac{1-\delta}{2}-\frac{5}{6} \delta}} \sum_{L} \frac{L^{\frac{1-\delta}{2}-\frac{\epsilon}{4}}}{\left\langle M^{2}+L\right\rangle^{\frac{1}{2}}} \lesssim \sum_{M \lesssim M_{1}} \frac{M^{s+1-\delta-\frac{\epsilon}{2}}}{\left\langle M_{1}\right\rangle^{s+1-\frac{8}{3} \delta}} \lesssim 1
$$

for $\epsilon=\frac{10}{3} \delta, s>-1+\frac{8}{3} \delta$, and

$$
\left\langle M_{2}\right\rangle^{-s} \lesssim N_{1}^{-s}
$$

for $s<0$, we get (3.18) for $-\frac{1}{2}<s<0$ by the same way as in the proof of Proposition 3.1. Case 1.

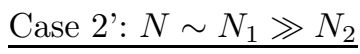


If $M \geq M_{1}$, then we have

$$
\langle M\rangle^{s}\left\langle M_{1}\right\rangle^{-s}\left\langle M_{2}\right\rangle^{-s} \lesssim\left\langle M_{2}\right\rangle^{-s} \lesssim N_{1}^{-s}
$$

for $s<0$. Therefore, we get (3.18) for $-\frac{1}{2}<s<0$ by the same way as in the proof of Proposition 3.1. Case 2.

While, if $M \leq M_{1}$, then we have

$$
J_{\delta, \epsilon}\left(N, M, N_{2}, M_{2}\right) \lesssim J_{\delta, \epsilon}\left(N_{1}, M_{1}, N_{2}, M_{2}\right) .
$$

Therefore, by estimating

$$
I \lesssim\left\|u_{N_{1}, M_{1}, L_{1}}\right\|_{L_{t x y}^{1-\delta}}\left\|v_{N_{2}, M_{2}, L_{2}} \cdot w_{N, M, L}\right\|_{L_{t x y}^{1+\delta}}
$$

instead of

$$
I \lesssim\left\|u_{N_{1}, M_{1}, L_{1}} \cdot v_{N_{2}, M_{2}, L_{2}}\right\|_{L_{t x y}^{\frac{2}{1+\delta}}}\left\|w_{N, M, L}\right\|_{L_{t x y}^{\frac{2}{1-\delta}}}
$$

in the proof of Proposition 3.1. Case 2, we get (3.18) for $-\frac{1}{2}<s<0$ by the same modification such as Case 1 '

Case 3': $N \sim N_{1} \sim N_{2} \geq 1$

If $\operatorname{supp} \mathcal{F}_{x, y}\left[w_{N, M, L}\right] \subset\{(\xi, \eta)|| \xi|\gg| \eta \mid$ or $|\xi| \ll|\eta|\}$, then $M \sim N$ holds. Therefore, we have

$$
\langle M\rangle^{s}\left\langle M_{1}\right\rangle^{-s}\left\langle M_{2}\right\rangle^{-s} \lesssim\langle N\rangle^{s}\left\langle N_{1}\right\rangle^{-s}\left\langle N_{2}\right\rangle^{-s} \lesssim N_{1}^{-s}
$$

for $s<0$ and get (3.18) for $-\frac{1}{2}<s<0$ by the same way as in the proof of Proposition 3.1. Case 3.

We assume $\operatorname{supp} \mathcal{F}_{x, y}\left[w_{N, M, L}\right] \subset\{(\xi, \eta)|| \xi|\sim| \eta \mid\}$. It suffice to show the estimate for $I_{1,2}$ and $I_{2,2}$, which are defined in Proposition 3.1. Case 3. By the same modification such as in Case 1', we can obtain (3.18) for $-\frac{1}{2}<s<0$.

\section{Acknowledgements}

This work is financially supported by JSPS KAKENHI Grant Number 17K14220 and Program to Disseminate Tenure Tracking System from the Ministry of Education, Culture, Sports, Science and Technology. The author would like to his appreciation to Shinya Kinoshita (Nagoya university) for his useful comments and discussions.

\section{References}

[1] M. B.-Artzi, H. Koch, and J.-C. Saut, Dispersion estimates for third order equations in two dimensions, Commun. Partial Differ. Equ 28 (2003), 19431974.

[2] D. Bekiranov, The initial value problem for the generalized Burgers' equation, Differ. Integral Equ. 9 (1996), 1253-1265.

[3] J. Bourgain, Fourier transform restriction phenomena for certain lattice subsets and applications to nonlinear evolution equations. I, II., Geom. Funct. Anal. 3 (1993), no. 3, 107-156, 209-262. 
[4] X. Carvajal, A. Esfahani, and M. Panthee, Well-posedness results and dissipative limit of high dimensional KdV-type equations, Bull Bras Math Soc, New Series 48 (2017), 505-550.

[5] M. Christ, J. Colliander, and T. Tao, Asymptotics, frequency modulation, and low regularity ill-posedness for canonical defocusing equations, Amer. J. Math. 125 (2003), 1235-1293.

[6] J. Colliander, M. Keel, G. Staffilani, H. Takaoka, and T. Tao, Sharp global well-posedness for $K d V$ and modified $K d V$ on $\mathbb{R}$ and $\mathbb{T}$, J. Amer. Math. Soc. 16 (2003), 705-749.

[7] D. B. Dix, Nonuniqueness and uniqueness in the initial-value problem for Burgers' equation, SIAM J. Math. Anal. 27 (1996), 708-724.

[8] J. Ginibre, Y. Tsutsumi, and G. Velo, On the Cauchy problem for the Zakharov system, J. Funct. Anal. 151 (1997), 384-436.

[9] A. Grünrock, and S. Herr, The Fourier restriction norm method for the Zakharov-Kuznetsov equation, Discrete. Contin. Dyn. Syst. 34 (2014), 20612068.

[10] Z. Guo, Global well-posedness of Korteweg-de Vries equation in $H^{-3 / 4}(\mathbb{R})$, J. Math. Pure. Appl. 91 (2009), 583-597.

[11] C. Kenig, G. Ponce, and G. Vega, A bilinear estimate with applications to the KdV equation, J. Amer. Math. Soc. 9 (1996), 573-603.

[12] C. Kenig, G. Ponce, and G. Vega, On the ill-posedness of some canonical dispersive equations, Duke. Math. J. 106 (2001), 617-633.

[13] N. Kishimoto, Well-posedness of the Cauchy problem for the Korteweg-de Vries equation at the critical regularity, Differ. Integral Equ. 22 (2009), 447-464.

[14] B. Kojok, Sharpe well-posedness for Kadomtsev-Petviashvili-Burgers (KPBII) equation in $\mathbb{R}^{2}$, J. Diff. Eqns 242 (2007), 211-247.

[15] N. A. Larkin, 2D Zakharov-Kuznetsov-Burgers equations on a strip, arXiv:1404.4638 1 [math.AP].

[16] N. A. Larkin, 2D Zakharov-Kuznetsov-Burgers equations with variable dissipation on a strip, Electron. J. Differential Equations, 2015 (2015), 1-20.

[17] F. Linares, and A. Pastor, Well-posedness for the two-dimensional modified Zakharov-Kuznetsov equation, SIAM J. Math. Anal. 41 (2009), 1323-1339.

[18] D. Mohamad, On the well-posedness for Kadomtsev-Petviashvili-Burgers I equation, J. Diff. Eqns 253 (2012), 1584-1603.

[19] L. Molinet, and D. Pilod, Bilinear Strichartz estimates for the ZakharovKuznetsov equation and applications, Ann. I. H. Poincaré - AN. 32 (2015), 347-371.

[20] L. Molinet, and F. Ribaud, On the low regularity of the Korteweg-de VriesBurgers equation, Int. Math. Res. Not. 37 (2002), 1979-2005. 
[21] L. Molinet, and S. Vento, Sharpe ill-posedness and well-posedness results for the KdV-Burgers equation: the real line case, Annali della Scuola Normale Superiore di Pisa, Classe di Scienze, 2011, 10 (3), pp.531-560. (hal00436652v2)

[22] W. M. Moslem, and R. Sabry, Zakharov-Kuznetsov-Burgers equation for dust ion acoustic waves, Chaos, Solitons and Fractals 36 (2008), 628-634.

[23] F. Ribaud, Cauchy problem for semilinear parabolic equations with initial data in $H_{p}^{s}\left(\mathbb{R}^{n}\right)$ spaces, Rev. Mat. Iberoamericana 14 (1998), no. 1, 1-46.

[24] H. L. Zhen, B. Tian, H. Zhong, W. R. Sun, and M. Li, Dynamics of the Zakharov-Kuznetsov-Burgers equations in dusty plasmas, Physics of Plasmas 20 (2013), doi:http://dx.doi.org/10.1063/1.4818508. 